\title{
Design of a Simple Orthogonal Multiwavelet Filter by Matrix Spectral Factorization
}

\author{
Vasil Kolev · Todor Cooklev · Fritz Keinert
}

Received: date / Accepted: date

\begin{abstract}
We consider the design of an orthogonal symmetric/antisymmetric multiwavelet from its matrix product filter by matrix spectral factorization (MSF). As a test problem, we construct a simple matrix product filter with desirable properties, and factor it using Bauer's method, which in this case can be done in closed form. The corresponding orthogonal multiwavelet function is derived using algebraic techniques which allow symmetry to be considered. This leads to the known orthogonal multiwavelet SA1, which can also be derived directly. We also give a lifting scheme for SA1, investigate the influence of the number of significant digits in the calculations, and show some numerical experiments.
\end{abstract}

Keywords orthogonal multiwavelets - matrix spectral factorization · Bauer's algorithm · Youla and Kazanjian algorithm · lifting scheme · PLUS matrices · Alpert multiwavelet

\section{Introduction and Problem Formulation}

A digital filter bank is a collection of $r$ filters $\mathbf{F}_{k}(z), k=0, \ldots, r-1$, which split a discretetime signal into $r$ subbands. These subband signals are usually decimated by a factor of $r$.

Vasil Kolev

Institute of Information and Communication Technologies

Bulgarian Academy of Sciences

B1. 2 Acad. G. Bonchev St.

1113 Sofia, Bulgaria

E-mail: kolev_acad@abv.bg

Todor Cooklev

Wireless Technology Center

Purdue University

Fort Wayne, IN 46805, USA

E-mail: cooklevt@pfw.edu

Fritz Keinert

Dept. of Mathematics

Iowa State University

Ames, IA 50011, USA

E-mail: keinert@iastate.edu 
Such a system is called a maximally decimated analysis bank. It can be used in applications such as signal, image, and video coding. For example, a scalar wavelet system is based on a single scaling function and mother wavelet, so $r=2$ [63]. In multiwavelet theory there are several scaling functions and mother wavelets [18]. This provides more degrees of freedom in the design, and makes it possible to simultaneously achieve several useful properties, such as symmetry, orthogonality, short support, and a higher number of vanishing moments [64].

The first multiscaling function (GHM) was constructed in 1994 by Geronimo, Hardin, and Massopust [37], based on fractal interpolation. The multiwavelet function with which the GHM multiscaling function forms a perfect reconstruction pair was constructed in a separate paper by Strang and Strela [65].

Cooklev et al. [18] investigated the general theory of $2 \times 2$ multifilter banks, and showed that real orthonormal multifilter banks and multiwavelets can be obtained from orthonormal complex-coefficient scalar filter banks. This ensures a number of good properties, such as orthogonality, regularity, symmetry, and a lattice-structure implementation. However, the limit functions in this case tend to have long support.

The design of multifilter banks and multiwavelets remains a significant problem. In the scalar case, spectral factorization of a half-band filter that is positive definite on the unit circle $\mathbb{T}$ in the complex plane was, in fact, the first design technique for perfect reconstruction filter banks, suggested by Smith and Barnwell [59]. This provides motivation to extend Cooklev's original idea [17] to the multiwavelet case, where spectral factorization is more challenging.

We consider a new approach for obtaining an orthogonal multiwavelet which possesses symmetry/antisymmetry and regularity, from a matrix product filter. This immediately leads to three main problems:

(A) How can we obtain an appropriate matrix product filter which satisfies strong requirements?

(B) How do we perform Matrix Spectral Factorization (MSF)?

(C) How do we improve the accuracy of the computed factors?

We will consider these three problems in more detail, as well as giving a brief description of some basic results.

Based on the previous theory and many applications of the SA1 multiwavelet, it is useful to develop a lifting scheme for it. Many of the above applications can then be implemented in software or hardware to be faster and more applicable.

The paper is organized as follows. After a brief review of multiwavelet background, we begin by constructing a half-band product filter with good smoothness properties. We then find a multiscaling function by using matrix spectral factorization. To complete the design by finding a multiwavelet function, we use additional algebraic techniques based on QR decomposition. The resulting multiwavelet is called SA1. It has orthogonality, symmetry/antisymmetry, piecewise smooth functions, and short support. We then construct a lifting scheme. Finally, some experiments are shown with respect to this simple multiwavelet. This includes investigating the influence of the number of significant digits used in the calculations, as well as the performance of the SA1 multiwavelet in terms of coding gain.

The main novel contributions of this paper are the following:

1. We introduce for the first time the product multifilter approach for designing a general MIMO filter bank;

2. We obtain for the first time an orthogonal multiscaling function by using the closed form of the matrix spectral factorization algorithm of Youla and Kazanjian [82]; 
3. We obtain the complementary orthogonal multiwavelet function;

4. We construct a lifting scheme for the obtained simple orthogonal multiwavelet;

5. We investigate the influence of the number of significant digits used for the coefficient $\sqrt{3}$, in $1 \mathrm{D}$ and $2 \mathrm{D}$ signal processing.

\section{Multiwavelet Theory}

We use the following notation conventions, illustrated with the letter 'a':

$a$ - lowercase letters refer to scalars or scalar functions;

a - lowercase bold letters are vectors or vector-valued functions;

$A$ - uppercase letters are matrices;

A - uppercase bold letters are matrix-valued functions.

$I$ and 0 are identity and zero matrices of appropriate size. $A^{T}$ denotes the transpose of the matrix $A$, and $A^{*}$ is the complex conjugate transpose. We are only considering real matrices in this paper. However, the variable $z$ used in matrix polynomials lies on the unit circle $\mathbb{T}$ in the complex plane, so that $\bar{z}=z^{-1}$. Thus, the complex conjugate transpose of

$$
\mathbf{A}(z)=\sum_{k} A_{k} z^{k}
$$

is given by

$$
\mathbf{A}(z)^{*}=\sum_{k} A_{k}^{T} z^{-k}
$$

The inner product of two vector-valued functions $\mathbf{f}(t)$ and $\mathbf{g}(t)$ in the $r$-dimensional Hilbert space $L^{2}(\mathbb{R})^{r}$ of square integrable functions is given by

$$
\langle\mathbf{f}, \mathbf{g}\rangle=\int \mathbf{f}(t) \mathbf{g}(t)^{*} d t
$$

which is a matrix.

2.1 Multiwavelets and the Discrete Multiwavelet Transform

Multiscaling and multiwavelet functions are vectors of $r$ scaling functions $\boldsymbol{\varphi}(t)$ and $r$ wavelet functions $\boldsymbol{\psi}(t)$, respectively:

$$
\boldsymbol{\varphi}(t)=\left[\begin{array}{c}
\varphi_{0}(t) \\
\varphi_{1}(t) \\
\vdots \\
\varphi_{r-1}(t)
\end{array}\right], \quad \boldsymbol{\psi}(t)=\left[\begin{array}{c}
\psi_{0}(t) \\
\psi_{1}(t) \\
\vdots \\
\psi_{r-1}(t)
\end{array}\right]
$$

They belong to $L^{2}(\mathbb{R})^{r}$, and satisfy the matrix dilation equations [63]

$$
\begin{aligned}
\boldsymbol{\varphi}(t) & =\sqrt{2} \sum_{k=0}^{m} H_{k} \boldsymbol{\varphi}(2 t-k), \\
\boldsymbol{\psi}(t) & =\sqrt{2} \sum_{k=0}^{m} G_{k} \boldsymbol{\varphi}(2 t-k) .
\end{aligned}
$$


Multiscaling and multiwavelet functions together are referred to as a multiwavelet. All of the functions have support contained in the interval $[0, m]$ (see [79]).

We only consider orthonormal multiwavelets, where for any integer $\ell$,

$$
\begin{gathered}
\langle\boldsymbol{\varphi}(t), \boldsymbol{\varphi}(t-\ell)\rangle=\langle\boldsymbol{\psi}(t), \boldsymbol{\psi}(t-\ell)\rangle=\delta_{0 \ell} I, \\
\langle\boldsymbol{\varphi}(t), \boldsymbol{\psi}(t-\ell)\rangle=0 .
\end{gathered}
$$

Equivalently, the matrix coefficients $H_{k}$ and $G_{k}$ satisfy the orthonormality conditions

$$
\begin{gathered}
\sum_{k} H_{k} H_{k+2 \ell}^{T}=\sum_{k} G_{k} G_{k+2 \ell}^{T}=\delta_{0 \ell} I, \\
\sum_{k} H_{k} G_{k+2 \ell}^{T}=0 .
\end{gathered}
$$

In the Discrete Multiwavelet Transform (DMWT), the input signal $\mathbf{s}^{0}$, at level $j=0$, is a sequence of $r$-vectors in $\ell_{2}$. For example, in the 2-channel case,

$$
\mathbf{s}^{0}=\left\{\mathbf{s}_{k}^{0}\right\}_{k=-\infty}^{\infty}, \quad \mathbf{s}_{k}^{0}=\left[\begin{array}{c}
s_{0}^{0}[k] \\
s_{1}^{0}[k]
\end{array}\right], \quad \text { with } \sum_{k}\left\|\mathbf{s}_{k}^{0}\right\|^{2}<\infty .
$$

The DMWT of $\mathbf{s}^{0}$ consists of computing recursively, for levels $j=1, \ldots J$, the approximation signal

$$
\mathbf{s}^{j}=\left\{\mathbf{s}_{\ell}^{j}\right\}_{\ell=-\infty}^{\infty}, \quad \mathbf{s}_{\ell}^{j}=\sum_{k} H_{k-2 \ell} \mathbf{s}_{k}^{j-1}
$$

and the detail signal

$$
\mathbf{d}^{j}=\left\{\mathbf{d}_{\ell}^{j}\right\}_{\ell=-\infty}^{\infty}, \quad \mathbf{d}_{\ell}^{j}=\sum_{k} G_{k-2 \ell} \mathbf{s}_{k}^{j-1} .
$$

We call $\mathbf{s}_{\ell}^{j}$ the scaling function coefficients, and $\mathbf{d}_{\ell}^{j}$ the wavelet coefficients.

Because of orthogonality, the inverse can be computed iteratively in a straightforward way:

$$
\mathbf{s}_{k}^{j-1}=\sum_{\ell} H_{k-2 \ell}^{T} \mathbf{s}_{\ell}^{j}+\sum_{\ell} G_{k-2 \ell}^{T} \mathbf{d}_{\ell}^{j}
$$

The matrix filter coefficients $H_{k}, G_{k}$ completely characterize the multiwavelet filter bank.

We can also describe the filters and the wavelet coefficients of the signal by their symbols

$$
\mathbf{H}(z)=\sum_{k} H_{k} z^{-k}, \quad \mathbf{G}(z)=\sum_{k} G_{k} z^{-k}, \quad \mathbf{s}^{j}(z)=\sum_{k} \mathbf{s}_{k}^{j} z^{k}, \quad \mathbf{d}^{j}(z)=\sum_{k} \mathbf{d}_{k}^{j} z^{k} .
$$

In symbol notation, one step of DMWT and inverse DMWT is described by

$$
\begin{aligned}
\mathbf{s}^{j}\left(z^{2}\right) & =\frac{1}{2}\left[\mathbf{H}(z) \mathbf{s}^{j-1}(z)+\mathbf{H}(-z) \mathbf{s}^{j-1}(-z)\right], \\
\mathbf{d}^{j}\left(z^{2}\right) & =\frac{1}{2}\left[\mathbf{G}(z) \mathbf{s}^{j-1}(z)+\mathbf{G}(-z) \mathbf{s}^{j-1}(-z)\right], \\
\mathbf{s}^{j-1}(z) & =\mathbf{H}(z)^{*} \mathbf{s}^{j}\left(z^{2}\right)+\mathbf{G}(z)^{*} \mathbf{d}^{j}\left(z^{2}\right) .
\end{aligned}
$$

Decomposition and reconstruction of the DMWT for a scalar input signal $s$ is represented in fig. 1. Since $s$ is scalar-valued, it is necessary to vectorize it to produce $\mathbf{s}^{0}$. A prefiltering step $\mathscr{M}(z)$ is inserted at the beginning of the analysis filter bank. A corresponding postfilter $\mathscr{N}(z)$ is incorporated at the end, which is the inverse of the prefiltering step. 


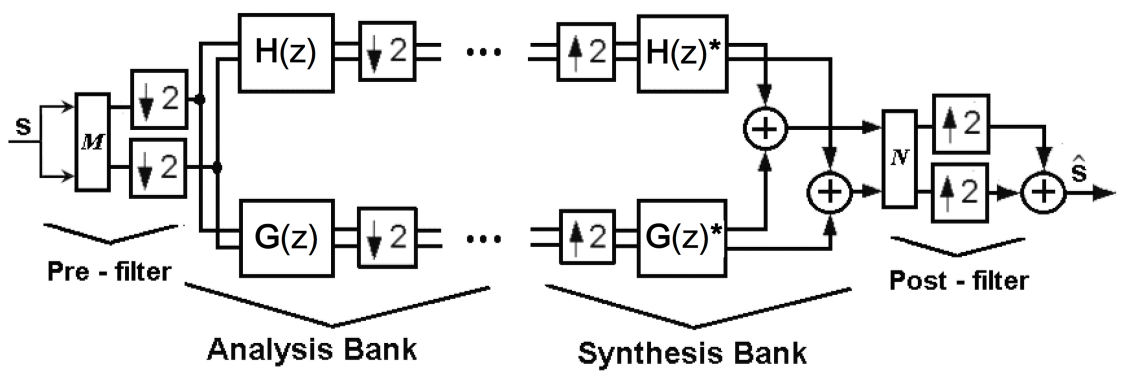

Fig. 1 Two-channel critically sampled analysis and synthesis filter banks, with prefilter $\mathscr{M}$ and postfilter $\mathscr{N}$ ( $\downarrow 2$ and $\uparrow 2$ represent decimation and zero-padding).

The result of executing the steps in eq. (6) is

$$
\hat{\mathbf{s}}^{0}(z)=\frac{1}{2}\left[\mathbf{H}(z)^{*} \mathbf{H}(z)+\mathbf{G}(z)^{*} \mathbf{G}(z)\right] \mathbf{s}^{0}(z)+\frac{1}{2}\left[\mathbf{H}(z)^{*} \mathbf{H}(-z)+\mathbf{G}(z)^{*} \mathbf{G}(-z)\right] \mathbf{s}^{0}(-z) .
$$

If we want perfect reconstruction (that is, $\hat{\mathbf{s}}^{0}=\mathbf{s}^{0}$ ), we need

$$
\begin{gathered}
\mathbf{H}(z)^{*} \mathbf{H}(z)+\mathbf{G}(z)^{*} \mathbf{G}(z)=2 I \\
\mathbf{H}(z)^{*} \mathbf{H}(-z)+\mathbf{G}(z)^{*} \mathbf{G}(-z)=0 .
\end{gathered}
$$

The orthogonality relations (2), expressed in terms of symbols, are

$$
\begin{gathered}
\mathbf{H}(z) \mathbf{H}(z)^{*}+\mathbf{H}(-z) \mathbf{H}(-z)^{*}=2 I, \\
\mathbf{G}(z) \mathbf{G}(z)^{*}+\mathbf{G}(-z) \mathbf{G}(-z)^{*}=2 I, \\
\mathbf{H}(z) \mathbf{G}(z)^{*}+\mathbf{H}(-z) \mathbf{G}(-z)^{*}=0
\end{gathered}
$$

It is easy to show that eqs. (7) and (8) are equivalent.

\subsection{Multiwavelet Denoising}

One of the most interesting signal processing applications is denoising. In most real applications the original signals are corrupted by noise. In addition, truncation error in the computation and in other processing introduces more errors. These errors can be interpreted as additive white Gaussian noise (AWGN) $e \sim N\left(0, \sigma^{2}\right)$ added to the original signal $s$. A denoising technique is a method of removing the noise while retaining important features of the signal. We consider denoising methods based on the multiwavelet transform.

The wavelet transform concentrates the energy of the true signal in a few wavelet coefficients, while the noise energy will be scattered among all coefficients. The wavelet coefficients of Gaussian noise are still Gaussian. Shrinking the small coefficients towards zero will eliminate most of the noise, while not affecting the signal very much.

We use the following notation. The original signal is $s$. This could be a one-dimensional signal $s(t)$, or an image $s(x, y)$. The noisy signal is $\tilde{s}=s+e$. The denoised signal is $\hat{s}$. We likewise use tildes and hats to denote the wavelet coefficients of $\tilde{s}, \hat{s}$. 


\subsubsection{Stochastic Sequences and Covariance Matrix}

Assume that the input is a noisy signal $\tilde{s}$. This is sampled and preprocessed into a vector sequence $\tilde{\mathbf{s}}^{0}$. The preprocessing is described by a linear operator $\mathscr{M}$. We also assume that postprocessing $\mathscr{N}$ is the inverse of preprocessing: $\mathscr{N} \mathscr{M}=I$. The discrete multiwavelet transform (DMWT) is described by a linear map $\Theta$. For orthogonal multiwavelet filter banks we have $\Theta^{T} \Theta=\Theta \Theta^{T}=I$.

It is important note the influence of the type of transform (orthonormal or biorthogonal) on the variance of stochastic sequences subjected to preprocessing and the DMWT. If the preprocessing $\mathscr{M}$ and $\Theta$ are orthonormal transforms, then the energy (i.e., the sum of squares of the elements) in the sequence $\mathscr{M} \tilde{\mathbf{s}}$ is unchanged: $\|\boldsymbol{\Theta} \mathscr{M} \tilde{\mathbf{s}}\|^{2}=\|\mathscr{M} \tilde{\mathbf{s}}\|^{2}=\|\tilde{\mathbf{s}}\|^{2}$. For biorthogonal transforms, both preprocessing and matrix transformation will change the input energy.

The noisy signal $\tilde{s}$ is the sum $\tilde{s}=s+e$, where $s$ is the true (deterministic) signal (noise free), and $e \sim N\left(0, \sigma^{2}\right)$ is the noise, with noise power $\sigma^{2}$. The goal of signal denoising is to minimize the mean square error MSE $=\frac{1}{N^{2}}\|s-\hat{s}\|$ subject to the condition that the denoised signal $\hat{s}$ is at least as smooth as $s$. Due to better signal representation by the multiwavelet transform, noise and signal can be separated much more easily. Multiwavelet denoising using a multivariate shrinkage operator effectively exploits the statistical information of the transformed wavelet coefficient vectors of noise, which improves the denoising performance. As a result of the decomposition and downsampling, the dependence of coefficients is reduced with increasing levels. The multiwavelet transform $\Theta$ with prefilter $\mathscr{M}$ of the noisy signal $\tilde{s}$

$$
\Theta \mathscr{M} \tilde{s}=\Theta \mathscr{M} s+\Theta \mathscr{M} e
$$

produces the vector wavelet coefficients

$$
\tilde{\mathbf{d}}_{k}^{j}=\mathbf{d}_{k}^{j}+\mathbf{f}_{k}^{j}
$$

which are correlated with their neighbors.

The scaling function coefficients $\mathbf{s}_{k}^{J}$ of the signal are fairly large, and do not need to be thresholded. We can concentrate on the wavelet coefficients $\mathbf{d}_{k}^{j}$ of the signal, and $\mathbf{f}_{k}^{j}$ of the noise.

The sequence $\mathbf{f}^{j}=\left\{\mathbf{f}_{k}^{j}\right\}$ of wavelet coefficients at level $j$ of the stochastic noise has a multivariate normal (Gaussian) distribution $\mathbf{f}^{j} \sim N\left(0, Y_{j}\right)$ with a covariance matrix

$$
\operatorname{cov}\left(\mathbf{f}^{j}\right)=\boldsymbol{\Theta}_{j} \mathscr{M}_{j} \Sigma \mathscr{M}_{j}^{T} \boldsymbol{\Theta}_{j}^{T},
$$

where $\Sigma$ is the covariance matrix of the noise. The matrices $Y_{j}$ are $r \times r$ blocks indicating the correlation between the vector coefficients inside each single decomposition level [4]. In the case of AWGN noise $e \sim N\left(0, \sigma_{e}^{2}\right)$,

$$
\operatorname{cov}\left(\mathbf{f}^{j}\right)=\boldsymbol{\Theta}_{j}\left[\sigma_{e}^{2} \mathscr{M} \mathscr{M}^{T}\right] \boldsymbol{\Theta}_{j}^{T}=\sigma_{e}^{2} \boldsymbol{\Theta}_{j}\left[\mathscr{M} \mathscr{M}^{T}\right] \boldsymbol{\Theta}_{j}^{T},
$$

the covariance of the stochastic part depends only on the preprocessing $\mathscr{M}$ and the choice of multiwavelet transform $\Theta$. If the preprocessing matrix satisfies $\mathscr{M}^{M}=I$, the covariance matrix is $\sigma_{e}^{2} I$. In the general case, $\operatorname{cov}\left(\mathbf{f}^{j}\right) \neq I$ is a symmetric block Toeplitz matrix.

Assuming that the blocks $Y_{j}$ describing the correlation between the vector coefficients are known, the nonnegative scalar values

$$
w_{k}^{j}=\sqrt{\left(\tilde{\mathbf{d}}_{k}^{j}\right)^{T} Y_{j}^{-1} \tilde{\mathbf{d}}_{k}^{j}}
$$

can be used for thresholding, as proposed [4]. In [28] it is shown that it is these values that should be thresholded, and the wavelet coefficient vectors can then be adapted accordingly. 


\subsubsection{Thresholding Rules}

There exist threshold and non-threshold wavelet shrinkage methods. A non-threshold wavelet shrinkage method is Besov shrinkage [22]21]. It is known that its scale is closed under real interpolation, and it is highly adaptive to the local smoothness.

In this paper, we consider threshold methods in more detail. This is a general wavelet denoising procedure [53,58]. Wavelet shrinkage denoising has been proven to be nearly optimal in the mean square error (MSE) sense, and performs well in simulation studies.

Thresholding methods can be grouped into two categories [61]: global thresholds and level-dependent thresholds. The first means that we choose a single value $\lambda$ for the threshold, to be applied globally to all wavelet coefficients; the second means that a possibly different threshold value $\lambda_{j}$ is chosen for each resolution level $j$. In what follows, we consider a global threshold $\lambda$.

The threshold distinguishes between insignificant coefficients attributed to noise, and significant coefficients that encode important signal features. Signals with sparse or near sparse representation, where only a small subset of the coefficients represent all or most of the signal energy, are fit for thresholding. The noise is estimated by a properly chosen threshold after the multiwavelet transform.

Filtering of additive Gaussian noise (zero mean and standard deviation $\sigma$ ) via the thresholding of wavelet coefficients was pioneered by Donoho [24]. A wavelet coefficient $\tilde{\mathbf{d}}_{k}^{j}$ is compared with a universal threshold $\lambda$. If the coefficient has a magnitude smaller than the threshold, it is considered to be noise, and is set to zero. Otherwise, is kept or modified, depending on the thresholding rule.

In this paper hard and soft thresholding methods will be considered. The vector wavelet coefficients thresholded by the soft thresholding rule are

$$
\hat{\mathbf{d}}_{k}^{j}= \begin{cases}\tilde{\mathbf{d}}_{k}^{j} \frac{w_{k}^{j}-\lambda}{w_{k}^{j}}, & w_{k}^{j} \geq \lambda, \\ 0, & w_{k}^{j}<\lambda .\end{cases}
$$

For the hard thresholding rule, they are

$$
\hat{\mathbf{d}}_{k}^{j}= \begin{cases}\tilde{\mathbf{d}}_{k}^{j}, & w_{k}^{j} \geq \lambda, \\ 0, & w_{k}^{j}<\lambda .\end{cases}
$$

Another method, semisoft thresholding, has been proposed [50,83], but so far only for the scalar wavelet setting.

The vector wavelet coefficients $\mathbf{d}_{k}^{j}$ could be thresholded using either the hard or soft thresholding rules. These thresholding rules have strong connections with model selection in the traditional linear models. The trick of thresholding is to include these irregularities while excluding the noise [27].

In general, in terms of visual quality, the noise reduction effect of soft thresholding is the best, followed by the improved semisoft shrinkage method; the hard thresholding method is the worst.

Hard shrinkage produces smaller bias but larger variance than soft shrinkage, and empirically performs better than soft shrinkage minimax estimation [10]. It better retains local characteristics of the image edges [24,66], and is suitable for denoising of noise with sudden changes. However, it introduces sudden jumps in the multiwavelet domain that in turn lead to oscillations in the reconstructed images. These oscillations are very close to the Gibbs 
phenomenon (see fig. 3 in [31]) and are called pseudo-Gibbs phenomena. They affect the visual quality of the denoising image, and manifest themselves as annoying artifacts in the denoised image (see Fig. 2/a)).

The soft thresholding method smoothes the jumps in the wavelet domain by shrinking also the coefficients near the jumps. The denoised signals end up relatively smooth, and the pseudo-Gibbs phenomenon is reduced to some extent.

Better results are expected in the future by using the multiple description approach for the multiwavelet transformation shown in [77].

\subsubsection{Universal Threshold}

The threshold value $\lambda$ is a very important parameter, which directly influences the performance of wavelet denoising. It can be determined by estimating the variance of the noise in images, but thise value is often unknown in applications.

Using a universal threshold value, instead of a different threshold at each level, is attractively simple, but is strictly suitable only when thresholding AWGN wavelet coefficients of variance $\sigma^{2}$ and mean zero. The basic idea is that if the signal component is in fact zero, then with high probability the combination of (zero) signal plus noise should not exceed the threshold level $\lambda=\sigma_{e} \sqrt{2 \log N}$, where $N$ is the length of the signal. The universal threshold $\lambda$ [24 27] pays attention to smoothness rather than to minimizing the mean square error (MSE).

This threshold is the asymptotic infimum, the asymptotic optimal threshold [25], and comes close to the minimax threshold, i.e. the threshold that minimizes the worst case MSE [26]. The trivial extension of the universal threshold to multiple wavelet coefficients is to use the threshold above applied to each coefficient element.

In the numerical examples in this paper, we are using the following Algorithm 1. It implements the algorithm described in the text, adapted to two-dimensional signals (images).

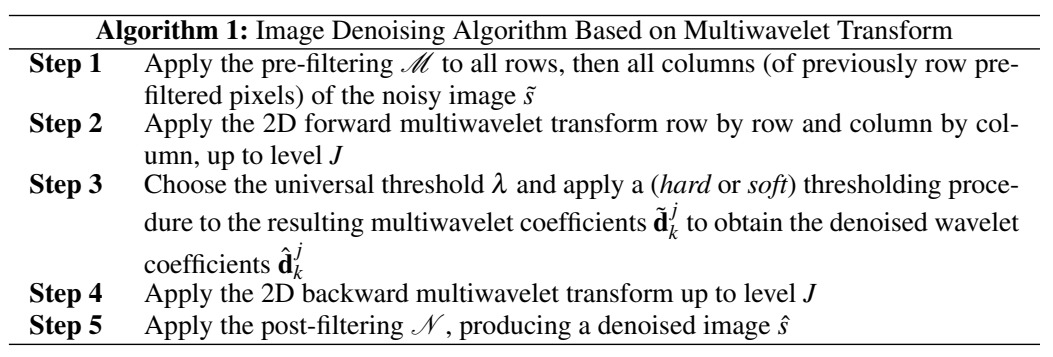

\subsection{The Multiwavelet SA1}

In this section, we describe a simple multiwavelet that will be used in the remainder of this paper to illustrate our approach. We are interested in supercompact multiwavelets, that is, multiwavelets with support in $[0,1]$. Such functions have many advantages:

- Application to functions defined on a finite interval does not require any special treatment at the boundaries of the interval [7];

- Interpolation with non-equally spaced data is possible [20,41];

- Application to functions that are only piecewise continuous (internal boundaries) can be efficiently implemented [7, 48, 51]; 


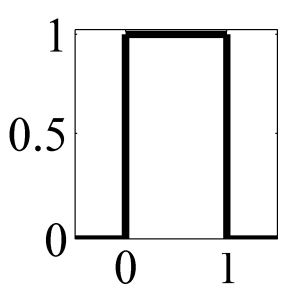

(a)

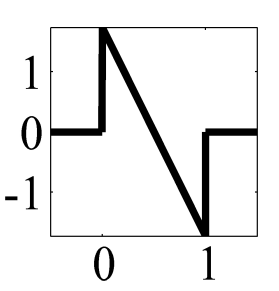

(b)

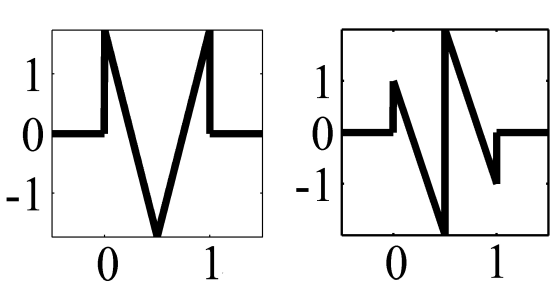

Fig. 2 The scaling and wavelet functions of SA1; (a) the multiscaling function $\boldsymbol{\varphi}(t)=\left[\varphi_{0}(t), \varphi_{1}(t)\right]^{T}$; (b) the multiwavelet function $\boldsymbol{\psi}(t)=\left[\psi_{0}(t), \psi_{1}(t)\right]^{T}$.

- It is easy to construct a nodal basis with (spectral) finite elements [5],55];

- Polynomials are reconstructed exactly [7];

- Discontinuities in the signal are easy to detect [80], and the size of jumps at element boundaries can be measured, in 1D [74] and 2D [75];

- Construction of the operation matrices in numerical methods is simple [1,23];

- Supercompact multiwavelets with the vanishing moment property are suitable for adaptive solvers of PDEs subject to boundary conditions, as well as for solving of a wide class of integro-differential operators having sparse representation in these bases.

Alpert [2] already observed that a multiscaling function whose entries form a basis of polynomials of degree $p$ on $[0,1]$ will always be refinable. For $p=0$, this leads to the Haar wavelet. For $p=1$, we can take $\phi_{0}=$ Haar wavelet, and $\phi_{1}=$ any non-constant linear polynomial. If we want $\phi_{1}$ to be orthogonal to $\phi_{0}$ and normalized, we find that for $t \in[0,1]$,

$$
\begin{aligned}
& \phi_{0}(t)=1, \\
& \phi_{1}(t)=\sqrt{3}(1-2 t) .
\end{aligned}
$$

This choice is unique, up to sign. The two components of $\boldsymbol{\varphi}$ are shown in fig. 2(a).

The refinement coefficients are easy to derive with elementary algebra: we find

$$
H_{0}=\frac{\sqrt{2}}{4}\left[\begin{array}{cc}
2 & 0 \\
\sqrt{3} & 1
\end{array}\right], \quad H_{1}=\frac{\sqrt{2}}{4}\left[\begin{array}{cc}
2 & 0 \\
-\sqrt{3} & 1
\end{array}\right] .
$$

The completion with a corresponding multiwavelet function is not unique, but by imposing orthogonality and symmetry constraints we find an essentially unique completion with recursion coefficients

$$
G_{0}=\frac{\sqrt{2}}{4}\left[\begin{array}{cc}
0 & 2 \\
-1 & \sqrt{3}
\end{array}\right], \quad G_{1}=\frac{\sqrt{2}}{4}\left[\begin{array}{ll}
0 & -2 \\
1 & \sqrt{3}
\end{array}\right] .
$$

Details are given in section 4.2 The corresponding functions are described by

$$
\begin{aligned}
& \psi_{0}(t)= \begin{cases}\sqrt{3}(1-4 t) & t \in\left[0, \frac{1}{2}\right], \\
\sqrt{3}(4 t-3) & t \in\left[\frac{1}{2}, 1\right],\end{cases} \\
& \psi_{1}(t)= \begin{cases}(1-6 t) & t \in\left[0, \frac{1}{2}\right], \\
(5-6 t) & t \in\left[\frac{1}{2}, 1\right] .\end{cases}
\end{aligned}
$$

They are shown in fig. 2 (b).

We call this multiwavelet SA1. 


\section{Matrix Product Filters}

3.1 Theory of Matrix Product Filters

Given a matrix filter

$$
\mathbf{H}(z)=\sum_{k=0}^{m} H_{k} z^{-k}
$$

its matrix product filter $\mathbf{P}(z)$ is defined as

$$
\mathbf{P}(z)=\mathbf{H}(z) \mathbf{H}(z)^{*}=\sum_{k=-m}^{m} P_{k} z^{k},
$$

where

$$
P_{k}=H_{0} H_{k}^{T}+\cdots+H_{m-k-1} H_{m-1}^{T}+H_{m-k} H_{m}^{T} .
$$

In particular for $k=0$,

$$
P_{0}=H_{0} H_{0}^{T}+\cdots+H_{m-1} H_{m-1}^{T}+H_{m} H_{m}^{T} .
$$

The Laurent polynomial matrix $\mathbf{P}(z) \in \mathbb{C}^{r \times r}\left[z, z^{-1}\right]$ is a discrete-time para-Hermitian matrix. Here $\mathbb{C}^{r \times r}\left[z, z^{-1}\right]$ is the ring of $r \times r$ matrices whose elements are polynomials in $z$, $z^{-1}$ with complex coefficients [9]. By the first equation in (8), it is a half-band filter [18], which means it satisfies the equation

$$
\mathbf{P}(z)+\mathbf{P}(-z)=2 I .
$$

This implies

$$
\begin{aligned}
P_{0} & =I, \\
P_{2 k} & =0 \quad \text { for } k \neq 0 .
\end{aligned}
$$

We can similarly define the half-band filter

$$
\mathbf{Q}(z)=\mathbf{G}(z) \mathbf{G}(z)^{*} .
$$

Every orthogonal multiscaling filter $\mathbf{H}(z)$ is a spectral factor of some half-band multifilter. We use this fact as the starting point for constructing multiwavelets: Construct a suitable $\mathbf{P}(z)$ first, and factor it to obtain $\mathbf{H}(z)$.

In the scalar case, spectral factorization is one of the main design techniques [8,63]. For $r>1$, matrix spectral factorization is required, which is more challenging.

\subsection{Matrix Spectral Factorization}

A critical component in classical spectral decomposition is the Fejér-Riesz theorem for positive definite functions [13]. Fejér [34] was the first to note the importance of the class of trigonometric polynomials that assume only non-negative real values; the theorem was considered and proved by Riesz [52].

The Fejér-Riesz theorem in one dimension considers a trigonometric polynomial expressed in the form

$$
v(z)=\sum_{k=-N}^{N} v_{k} z^{k} .
$$


When $v(z)$ is real for all $z \in \mathbb{T}$, the coefficients satisfy $\overline{v_{k}}=v_{-k}$ for all $k$. The theorem states that if $v(z) \geq 0$ for all $z \in \mathbb{T}$, such a $v(z)$ is expressible in the form

$$
v(z)=p(z) p(z)^{*}
$$

for some polynomial $p(z)=\sum_{k=0}^{N} p_{k} z^{k}$. As noted in [32], a spectral factor $p(z)$ is unique up to a constant right unitary multiplier $U(z)$, i. e.

$$
p_{\text {new }}(z)=p(z) U(z) .
$$

The Fejér-Riesz theorem does not extend to factorization of multivariate trigonometric polynomials (see [29] for some counterexamples). Relevant key theorems are [54, Theorem 3.2] in the 1D case, [29, Theorem 6.2] for the 2D case, with examples and necessary and sufficient conditions in [38], and [54, Theorem 3.1] for arbitrary dimensions.

However, the theorem can be extended to the case of univariate polynomials with matrix coefficients. This is Matrix Spectral Factorization (MSF) (see [73, 12,33,82]).

The MSF problem plays a crucial role in the solution of various applied problems for MIMO systems in communications and control engineering [9, 45, 78, 81].

\subsection{Bauer's Method}

A well-known method for MSF is Bauer's method [6]. This method has been successfully applied in [14,30,35,39]. Details of the algorithm are given in section 4.1 below.

Bauer's method, in the implementation of Youla and Kazanjian [47,46 49,82], has the advantage over other approaches that it can handle zeros of the determinant of $\mathbf{P}(z)$ on $\mathbb{T}$. Unfortunately, the presence of these zeros affects the accuracy and speed of convergence. In its original form, the method only gives us a low precision spectral factor, and convergence is very slow.

The approaches in [33] for finding precision spectral factors can be used instead. For instance, the Janashia-Lagvilava MSF method for the orthogonal SA4 multiwavelet considered in [33] is exact.

In this paper, we achieve an exact MSF leading to a simple orthogonal multiwavelet by the closed form of Bauer's method for short product filters. This is possible for some types of short support multiwavelets, especially the supercompact multiwavelets [7].

\subsection{A Simple Matrix Product Filter}

The simplest non-scalar example is for $r=2, m=1$. In this case, $\mathbf{P} \in C^{2 \times 2}\left[z, z^{-1}\right]$ has the form

$$
\mathbf{P}(z)=P_{1}^{T} z^{-1}+P_{0}+P_{1} z
$$

From the half-band condition (11) we see that $P_{0}$ is the identity matrix.

The smoothness of the multiscaling and multiwavelet functions is improved if the determinant of the product filter has a higher-order zero at $z=-1$ (see [72]). Therefore, we choose

$$
\operatorname{det}(\mathbf{P}(z))=\left(\frac{1+z^{-1}}{2}\right)^{k} q(z),
$$

where $q(z)$ is a linear phase polynomial in $z$, and $k$ is necessarily even. 
The simplest case corresponds to $k=4$ and $q(z)=z^{2}$, where

$$
\operatorname{det}(\mathbf{P}(z))=\left(\frac{1+z^{-1}}{2}\right)^{4} z^{2}=\frac{1}{16}\left(z^{-2}+4 z^{-1}+6+4 z+z^{2}\right) .
$$

Setting

$$
P_{1}=\left[\begin{array}{ll}
a & b \\
c & d
\end{array}\right]
$$

we find the equations

$$
\begin{aligned}
a d-b c & =1 / 16, \\
a+d & =4 / 16, \\
1-b^{2}-c^{2}+2 a d & =6 / 16 .
\end{aligned}
$$

These equations have precisely four solutions: either $a=1 / 2, d=-1 / 4$ or $a=-1 / 4$, $d=1 / 2$, and in both cases we can choose $b=-c= \pm \sqrt{3} / 4$. However, these are all essentially equivalent. The different choices just correspond to interchanging $\phi_{0}$ and $\phi_{1}$, or changing the sign of one of the functions.

We choose

$$
a=\frac{1}{2}, \quad b=-\frac{\sqrt{3}}{4}, \quad c=\frac{\sqrt{3}}{4}, \quad d=-\frac{1}{4} .
$$

The resulting product filter has coefficient

$$
P_{1}=\frac{1}{4}\left[\begin{array}{cc}
2 & -\sqrt{3} \\
\sqrt{3} & -1
\end{array}\right] .
$$

\section{Design of Orthogonal Multiwavelet Filter}

\subsection{Finding $\mathbf{H}(z)$}

For simplicity, we only describe the details of Bauer's method for the case $m=1$ considered in this paper. The equation $\mathbf{P}(z)=\mathbf{H}(z) \mathbf{H}(z)^{*}$ is equivalent to the fact that the infinite block tridiagonal matrix

$$
P=\left[\begin{array}{ccccccc}
\ddots & \ddots & \ddots & & & \\
& P_{1}^{T} & P_{0} & P_{1} & & \\
& & P_{1}^{T} & P_{0} & P_{1} & \\
& & & \ddots & \ddots & \ddots
\end{array}\right]
$$

can be factored as $P=H H^{T}$, where $H$ is the infinite block bidiagonal matrix

$$
H=\left[\begin{array}{lllllll}
\ddots & \ddots & & & & \\
& H_{1} & H_{0} & & \\
& & H_{1} & H_{0} & \\
& & & \ddots & \ddots
\end{array}\right]
$$

For a chosen integer $n$, we truncate $P$ to the matrix $P_{n}$ of size $(n+1) \times(n+1)$, and do a Cholesky factorization $P_{n}=H_{n} H_{n}^{T}$. The last row of $H_{n}$ will be $H_{1}^{(n)}, H_{0}^{(n)}$. 
The YK theory says that $H_{0}^{(n)} \rightarrow H_{0}$ as $n \rightarrow \infty$, and likewise for $H_{1}$. The computation can be streamlined, by defining

$$
X^{(k)}=H_{0}^{(k)}\left[H_{0}^{(k)}\right]^{T}, \quad k=0,1, \ldots
$$

The Cholesky factorization algorithm is equivalent to

$$
\begin{aligned}
X^{(0)} & =P_{0}, \\
X^{(k+1)} & =P_{0}-P_{1}^{T}\left[X^{(k)}\right]^{-1} P_{1} .
\end{aligned}
$$

The limit matrix $X$ satisfies

$$
X=P_{0}-P_{1}^{T} X^{-1} P_{1} .
$$

We can use fixed point iteration based on (15) to avoid forming the large matrix $P_{n}$. Once $X$ has been found to sufficient accuracy, we can find $H_{0}$ as the Cholesky factor of $X$, and $H_{1}=P_{1}^{T} H_{0}^{-T}$.

Even better, in the simple case considered in this paper, the Symbolic Math toolbox in Matlab is able to solve equation (16) in closed form. On a MacBook Air with 2.2GHz Intel i7 processor, using Matlab 9.1, the computational time is under $0.8 \mathrm{sec}$. We get the exact matrix spectral factor with coefficients

$$
H_{0}=\frac{\sqrt{2}}{4}\left[\begin{array}{cc}
2 & 0 \\
\sqrt{3} & 1
\end{array}\right], \quad H_{1}=\frac{\sqrt{2}}{4}\left[\begin{array}{cc}
2 & 0 \\
-\sqrt{3} & 1
\end{array}\right] .
$$

This is precisely the multiscaling function of SA1, introduced in section 2.3

The spectral factor obtained by our closed form of Bauers method is precise. This multiscaling function can also be obtained from the $4 \times 4$ Type-II DCT matrix [36] and by using the Janashia-Lagvilava method [33] in exact form. It can also be obtained approximately by the Janashia-Lagvilava matrix spectral factorization method (see procedure 2 in [42]), with an error in the spectral factors of about $10^{-8}$.

\subsection{Finding $\mathbf{G}(z)$}

The orthogonality conditions $(2)$ in the case $m=1$ simply state that the matrix

$$
\left[\begin{array}{ll}
H_{0} & H_{1} \\
G_{0} & G_{1}
\end{array}\right]
$$

must be orthogonal. We can find an initial orthogonal completion $\left[\hat{G}_{0}, \hat{G}_{1}\right]$ from a QRdecomposition of $\left[H_{0}, H_{1}\right]^{T}$. Matlab returns

$$
\left[\hat{G}_{0}, \hat{G}_{1}\right]=\frac{\sqrt{2}}{4}\left[\begin{array}{cccc}
1 & -\sqrt{3} & -1 & -\sqrt{3} \\
0 & 2 & 0 & -2
\end{array}\right] .
$$

Any other orthogonal completion has to be of the form

$$
\left[G_{0}, G_{1}\right]=U \cdot\left[\hat{G}_{0}, \hat{G}_{1}\right]
$$

for orthogonal $U$. We want to choose a $U$ that introduces symmetry. 
A function $f(t)$ is symmetric about a point $a$ if $f(a+t)=f(a-t)$. It is antisymmetric about $a$ if $f(a+t)=-f(a-t)$. For multiscaling and multiwavelet functions, we only consider the simplest case, where each component $\varphi_{i}$ or $\psi_{i}$ is symmetric or antisymmetric about the midpoint $m / 2$. Thus,

$$
\boldsymbol{\varphi}\left(\frac{m}{2}+t\right)=S \cdot \boldsymbol{\varphi}\left(\frac{m}{2}-t\right),
$$

where $S$ is a diagonal matrix with entries 1 (symmetry) or $(-1)$ (antisymmetry). Likewise,

$$
\boldsymbol{\psi}\left(\frac{m}{2}+t\right)=T \cdot \boldsymbol{\psi}\left(\frac{m}{2}-t\right)
$$

It is not hard to show that $\boldsymbol{\varphi}, \boldsymbol{\psi}$ have the symmetry given by $S, T$ if and only if

$$
\begin{aligned}
& H_{k}=S H_{m-k} S, \\
& G_{k}=T G_{m-k} S, \quad k=0, \ldots, m .
\end{aligned}
$$

In our example, we observe that the original factors 17 already satisfy

$$
H_{1}=S H_{0} S
$$

for $S=\operatorname{diag}(1,-1)$. That is, component $\varphi_{0}$ is symmetric, $\varphi_{1}$ is antisymmetric.

The symmetry conditions $(19)$ for $\mathbf{G}$ are

$$
\begin{aligned}
& U \hat{G}_{0}=T U \hat{G}_{1} S, \\
& U \hat{G}_{1}=T U \hat{G}_{0} S,
\end{aligned}
$$

which leads to

$$
U=T U \hat{G}_{1} S \hat{G}_{0}^{-1}=-T U S .
$$

It is easy to check that $T= \pm I$ is not possible, so $T= \pm S$. We choose $T=S$, which means that we want $\psi_{0}$ to be symmetric, $\psi_{1}$ antisymmetric.

Any $U$ of the form

$$
U=\left[\begin{array}{cc}
0 & \pm 1 \\
\pm 1 & 0
\end{array}\right]
$$

is then suitable. The choice of signs corresponds to changing the sign of $\psi_{0}$ and/or $\psi_{1}$. Using

$$
U=\left[\begin{array}{cc}
0 & 1 \\
-1 & 0
\end{array}\right]
$$

leads to the same $G_{0}, G_{1}$ as in 10 .

Choosing $T=-S$ would interchange $\psi_{0}$ and $\psi_{1}$, and give us

$$
U=\left[\begin{array}{cc} 
\pm 1 & 0 \\
0 & \pm 1
\end{array}\right]
$$

which again corresponds to sign changes. 
(a)

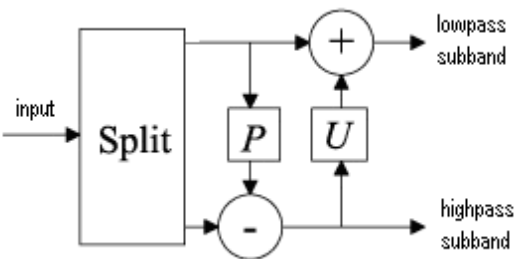

(b)

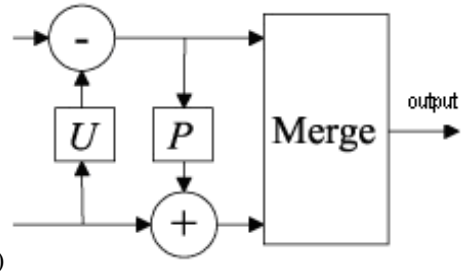

Fig. 3 One lifting step; (a) analysis part; (b) synthesis part.

\section{Efficient Implementation}

We give here a brief overview of the lifting scheme (LS) and some rounding operations. Lifting steps are an efficient implementation of filtering operations (see fig. 3. 3 )). One lifting step for a filter bank consists of an operation Split, a prediction $P$, and an update $U$.

One of the great advantages of LS is that it decomposes a multiwavelet filter into simple invertible steps [11 67]. The inverse LS can be obtained from the forward LS by reversing the order of the operations, inverting the signs in the lifting steps, and replacing the Split by a Merge step (see fig. 3(b)).

If a floor (or round or ceiling) operator is placed in each lifting step, the filter banks can now map integers to integers with perfect reconstruction, regardless of whether the lifting step has integer coefficients or not.

Moreover, if the factors are chosen to be dyadic, multiplication can be implemented by addition and binary bit shift. If the integer mantissa $k$ in a lifting step with coefficient $c=k \cdot 2^{-b_{0}}$ is written in binary notation as

$$
k=\sum k_{i} 2^{i}, \quad k_{i}=0 \text { or } 1,
$$

then the multiplication of a number $x$ by $c$ can be implemented by adding copies of $x$, leftshifted by $i$, for each $k_{i} \neq 0$, and a final right-shift by $b_{0}$ bits.

This approach leads to an implementation with solely binary shifts, preventing bit-depth expansion in intermediate results. With all scaling factors set to unity, multiplierless filter banks can be easily constructed using this method. If the scaling factors are powers of two, we can still retain the multiplierless feature on filter banks.

The lifting scheme can be done in place: we never need samples other than the output of the previous lifting step, and therefore we can replace the old stream by the new stream at every summation point.

\subsection{The Lifting Scheme}

Based on matrix factorization theory [40], a nonsingular matrix can be factored into a product of at most three triangular elementary PLUS reversible matrices [56, 76, 44]. If the diagonal elements of the matrices are ones, a reversible integer-to-integer transform can be realized by LS.

In order to speed up the implementation, it is necessary to optimize the elementary triangular factorization by either minimizing the number of factorized matrices or the computational complexity of each step. The factorization is not unique, and there are other different factorizations that affect the error between the dyadic approximation transform and the original transform. 
(a)
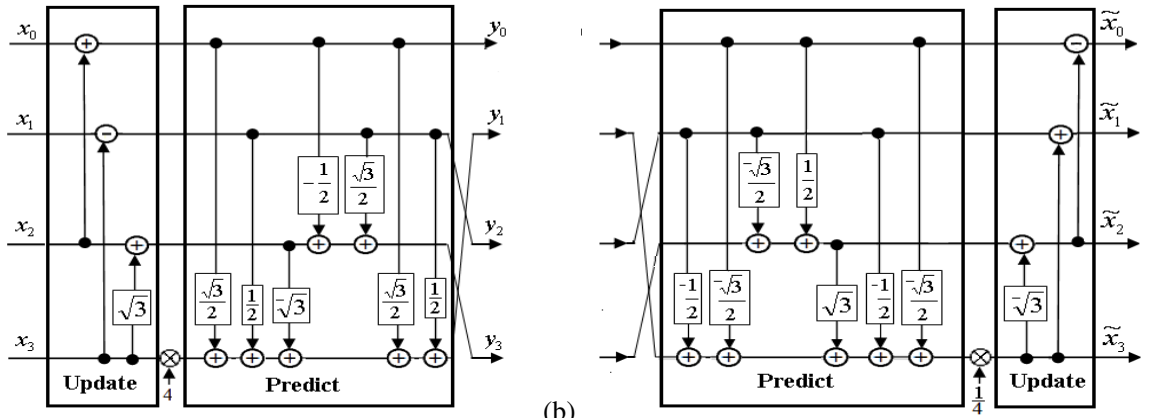

Fig. 4 The PLUS matrices implementation of $H$; (a) forward; (b) backward.

For input vector $\mathbf{x}=\left[x_{0}, x_{1}, x_{2}, x_{3}\right]^{T}$ and output vector $\mathbf{y}=\left[y_{0}, y_{1}, y_{2}, y_{3}\right]^{T}$, the lifting implementation of the forward DMWT [6] is

$$
\mathbf{y}=H \mathbf{x}=(P L U) \mathbf{x}=\left[\begin{array}{llll}
1 & 0 & 0 & 0 \\
0 & 0 & 0 & 1 \\
0 & 1 & 0 & 0 \\
0 & 0 & 1 & 0
\end{array}\right]\left[\begin{array}{cccc}
1 & 0 & 0 & 0 \\
0 & 1 & 0 & 0 \\
-\frac{1}{2} & \frac{\sqrt{3}}{2} & 1 & 0 \\
\frac{\sqrt{3}}{2} & \frac{1}{2} & -\sqrt{3} & 1
\end{array}\right]\left[\begin{array}{cccc}
1 & 0 & 1 & 0 \\
0 & 1 & 0 & -1 \\
0 & 0 & 1 & \sqrt{3} \\
0 & 0 & 0 & 4
\end{array}\right]\left[\begin{array}{l}
x_{0} \\
x_{1} \\
x_{2} \\
x_{3}
\end{array}\right]
$$

This leads to the synthesis implementation

$$
H^{-1}=\frac{1}{2} U^{-1} L^{-1} P^{T} .
$$

We include the factor of $(1 / 2)$ with the reconstruction instead of the decomposition (see (6)).

In our implementation, we write the $L$ and $U$ matrices as products of elementary triangular and/or diagonal reversible matrices (see [62], page 34), for faster evaluation.

$$
\begin{aligned}
L & =\left[\begin{array}{cccc}
1 & 0 & 0 & 0 \\
0 & 1 & 0 & 0 \\
-1 / 2 & \sqrt{3} / 2 & 1 & 0 \\
0 & 0 & 0 & 1
\end{array}\right]\left[\begin{array}{cccc}
1 & 0 & 0 & 0 \\
0 & 1 & 0 & 0 \\
0 & 0 & 1 & 0 \\
\sqrt{3} / 2 & 1 / 2 & -\sqrt{3} & 1
\end{array}\right], \\
U & =\left[\begin{array}{llll}
1 & 0 & 0 & 0 \\
0 & 1 & 0 & 0 \\
0 & 0 & 1 & 0 \\
0 & 0 & 0 & 4
\end{array}\right]\left[\begin{array}{cccc}
1 & 0 & 0 & 0 \\
0 & 1 & 0 & -1 \\
0 & 0 & 1 & \sqrt{3} \\
0 & 0 & 0 & 1
\end{array}\right]\left[\begin{array}{cccc}
1 & 0 & 1 & 0 \\
0 & 1 & 0 & 0 \\
0 & 0 & 1 & 0 \\
0 & 0 & 0 & 1
\end{array}\right]
\end{aligned}
$$

The implementation of the lifting scheme is shown in fig. 4

5.2 Multiplierless architecture of the coefficient $\sqrt{3}$

For the transform to be reversible, it must be able to exactly reconstruct the input from the output. Low computational complexity is another desirable property. In order to avoid floating-point multiplications, we approximate values of the lifting matrices by dyadic numbers.

The lifting scheme described in subsection 5.1 uses only a single non-dyadic number, which is $\sqrt{3}$. We want to approximate this coefficient by a number of the form $k \cdot 2^{-b_{0}}$ with 
(a)

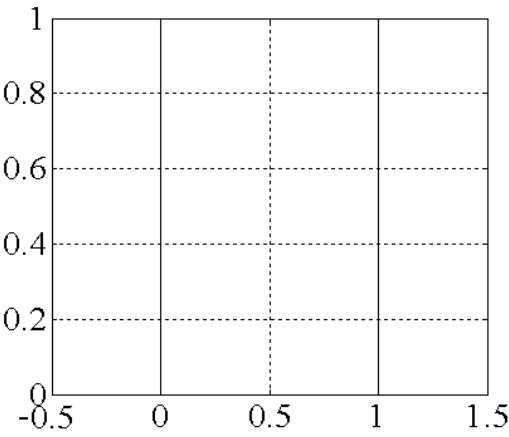

(c)

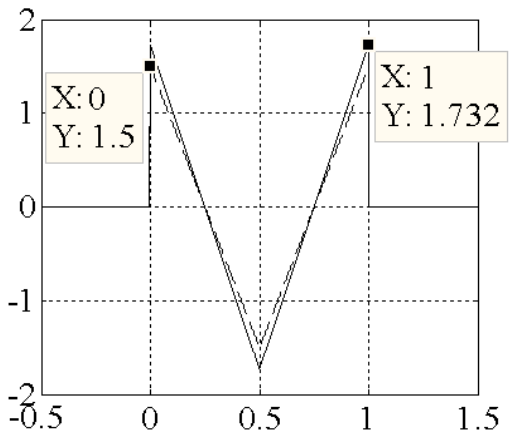

(b)

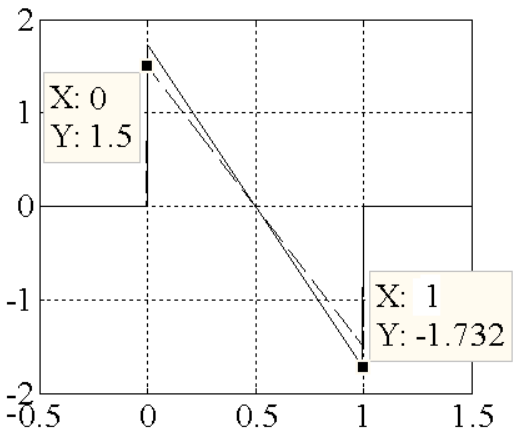

(d)

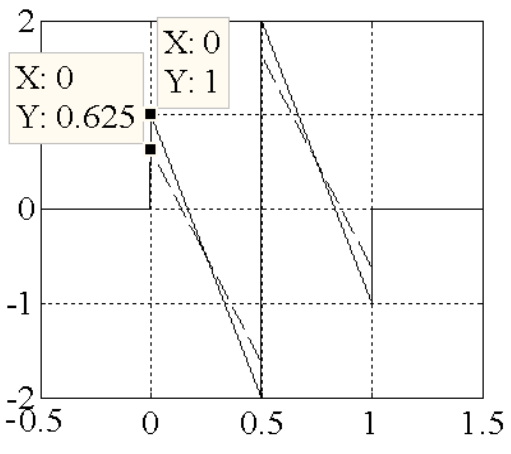

Fig. 5 Floating-point arithmetic (solid line) in comparison with fixed point arithmetic (dash-dot line) of the impulse responses of the SA1 orthogonal multiwavelet, with 2-bit dyadic approximation of the coefficient $\sqrt{3}$ (that is, $\sqrt{3} \approx 3 / 2$ ); (a) $\phi_{0}$; (b) $\phi_{1} ;$; (c) $\psi_{0} ;$; (d) $\psi_{1}$.

integer $k$. Since $\sqrt{3}$ is between 1 and 2 , this corresponds to an approximation with $\left(b_{0}+1\right)$ bits. For example, for $b_{0}=5, \sqrt{3}$ is approximated by the 6-bit number $55 \cdot 2^{-5}=1.71875$, with an error of approximately $1.73205-1.71875=0.0133$.

The dyadic approximations of the coefficient $\sqrt{3}$ and its quantization errors are shown in table 1 The table also shows the number of adders required to implement the multiplierless structure.

We illustrate the effect of quantization for $b_{0}=1$ in more detail. This is the case of 2-bit approximation, with $\sqrt{3} \approx 3 / 2$.

The true recursion coefficients of SA1 are given in eqs. (9) and (10). The actual recursion coefficients used in the 2-bit approximation are

$$
\begin{array}{cc}
\tilde{H}_{0}=\frac{\sqrt{2}}{4}\left[\begin{array}{cc}
2 & 0 \\
1.5 & 1
\end{array}\right], & \tilde{H}_{1}=\frac{\sqrt{2}}{4}\left[\begin{array}{cc}
2 & 0 \\
-1.5 & 1
\end{array}\right], \\
\tilde{G}_{0}=\frac{\sqrt{2}}{4}\left[\begin{array}{cc}
0 & 2 \\
-1 & 1.5
\end{array}\right], & \tilde{G}_{1}=\frac{\sqrt{2}}{4}\left[\begin{array}{ll}
0 & -2 \\
1 & 1.5
\end{array}\right] .
\end{array}
$$


Table 1 Different quantizations of the non-trivial coefficient $\sqrt{3} \approx k \cdot 2^{-b_{0}}$, with $k$ an integer represented by $\left(b_{0}+1\right)$ bits. For example, for $b_{0}=5$, the approximation is $\sqrt{3} \approx 55 \cdot 2^{-5}=1.71875$, with an error of approximately $1.73205-1.71875=0.0133$. The table also shows the number of adders needed to realize the multiplierless structure. Entries in bold mark the places where increasing the number of bits does not change the approximation.

\begin{tabular}{cccr}
\hline$b_{0}=$ binary exponent & mantissa & adders & \multicolumn{1}{c}{ quantization error } \\
\hline 1 & 3 & 1 & 0.232050807568877 \\
2 & 7 & 2 & -0.017949192431123 \\
$\mathbf{3}$ & $\mathbf{1 4}$ & $\mathbf{2}$ & ---- \\
$\mathbf{4}$ & $\mathbf{2 8}$ & $\mathbf{2}$ & ---- \\
5 & 55 & 2 & 0.013300807568877 \\
6 & 111 & 2 & -0.002324192431123 \\
$\mathbf{7}$ & $\mathbf{2 2 2}$ & $\mathbf{2}$ & ---- \\
8 & 443 & 3 & 0.001582057568877 \\
9 & 887 & 3 & -0.000371067431123 \\
$\mathbf{1 0}$ & $\mathbf{1 7 7 4}$ & $\mathbf{3}$ & ---- \\
11 & 3547 & 4 & 0.000117213818877 \\
$\mathbf{1 2}$ & $\mathbf{7 0 9 4}$ & $\mathbf{4}$ & ---- \\
13 & 14189 & 4 & -0.000004856493623 \\
$\mathbf{1 4}$ & $\mathbf{2 8 3 7 8}$ & $\mathbf{4}$ & ---- \\
\hline
\end{tabular}

These approximate matrix coefficients (22) satisfy

$$
\begin{gathered}
\tilde{\mathbf{H}}(z) \tilde{\mathbf{H}}(z)^{*}+\tilde{\mathbf{H}}(-z) \tilde{\mathbf{H}}(-z)^{*}=\left[\begin{array}{cc}
2 & 0 \\
0 & \frac{13}{8}
\end{array}\right], \\
\tilde{\mathbf{G}}(z) \tilde{\mathbf{G}}(z)^{*}+\tilde{\mathbf{G}}(-z) \tilde{\mathbf{G}}(-z)^{*}=\left[\begin{array}{cc}
2 & 0 \\
0 & \frac{13}{8}
\end{array}\right], \\
\tilde{\mathbf{H}}(z) \tilde{\mathbf{G}}(z)^{*}+\tilde{\mathbf{H}}(-z) \tilde{\mathbf{G}}(-z)^{*}=0
\end{gathered}
$$

instead of the true orthogonality conditions 8 .

This leads to a decrease of $\mathrm{CG}$ to $2.01 \mathrm{~dB}$ and non-perfect reconstruction, which is undesirable in many mathematical and engineering applications.

The effect of rounding can also be illustrated by its effect on the multiscaling and multiwavelet functions. It is easy to verify that the functions which satisfy the rounded recursion relations corresponding to (1) are

$$
\tilde{\phi}_{0}(t)=1, \quad \tilde{\phi}_{1}(t)=\frac{3}{2}(1-2 t)
$$

and

$$
\tilde{\psi}_{0}(t)=\left\{\begin{array}{ll}
\frac{3}{2}(1-4 t) & t \in\left[0, \frac{1}{2}\right], \\
\frac{3}{2}(4 t-3) & t \in\left[\frac{1}{2}, 1\right],
\end{array} \quad \tilde{\Psi}_{1}(t)=\left\{\begin{array}{lll}
\frac{5}{8}-\frac{9}{2} t & t \in\left[0, \frac{1}{2}\right] \\
\frac{31}{8}-\frac{9}{2} t & t \in\left[\frac{1}{2}, 1\right] .
\end{array}\right.\right.
$$

This is illustrated in fig. 5. The biggest differences are at the end points, which are marked by squares in the figure.

Obviously, the dyadic approximation strongly influences the sloped parts in time domain, which leads to smaller magnitudes for the scaling function $\tilde{\phi}_{1}$, as well as both wavelet functions $\tilde{\psi}_{0}$ and $\tilde{\psi}_{1}$. 
Table 2 Comparison of CG, symmetric/antisymmetric properties (SA), Sobolev regularity (S) and autocorrelation matrix $R_{x}$ of $\mathrm{AR}(1)$ and $\rho=0.95$.

\begin{tabular}{ccccc}
\hline Multiwavelet & CG & S & SA & Length \\
\hline Biorthogonal (dual to Hermitian cubic spline) ('bih32s') [3] & 1.01 & 2.5 & yes & $3 / 5$ \\
Biorthogonal Hermitian cubic spline ('bih34n') [66] & 1.51 & 2.5 & yes & $3 / 5$ \\
Integer Haar [15] & 1.83 & 0.5 & yes & 2 \\
CL [16] & 2.06 & 1.06 & yes & 3 \\
SA1 & 2.13 & 0.5 & yes & 2 \\
SA4 [57] & 3.73 & 0.99 & yes & 4 \\
GHM [37] & 4.41 & 1.5 & yes & 4 \\
\hline
\end{tabular}

\section{Performance Analysis}

\subsection{Comparative Analysis}

The objective of this section is to evaluate numerically the performance of the SA1 multiwavelet, and compare it to some well-known orthogonal and biorthogonal multiwavelets, with the criteria being coding gain (CG) [60]70], Sobolev smoothness (S) [43], symmetry/antisymmetry (SA), and length. The CG for orthogonal transforms is a good indication of the performance in signal processing. It is the ratio of arithmetic and geometric means of channel variances

$$
C G=\frac{\frac{1}{r} \sum_{i=1}^{r} \sigma_{i}^{2}}{\left(\prod_{i=1}^{r} \sigma_{i}^{2}\right)^{1 / r}} .
$$

Coding gain is one of the most important factors to be considered in many applications. A transform with higher coding gain compacts more energy into a smaller number of coefficients. A comparison of CGs is given in table 2 Sobolev regularity, symmetry/antisymmetry properties and the length of the given multiwavelets are also included. The CG is computed using a first order Markov model AR(1) with intersample autocorrelation coefficient $\rho=0.95[71]$. Obviously, despite the short length (only two matrix coefficients) of the simple multiwavelet SA1, its CG is better than that of most of the orthogonal and biorthogonal multiwavelets.

\subsection{Pre- and Postfilters}

In the case of a scalar-valued input signal to the multiple-input multiple-output (MIMO) filter bank it is necessary to vectorize the input. In other words, a pre-processing step (called prefiltering) must be inserted at the beginning of the analysis multifilter; in a symmetric way, a postfilter must be incorporated at the end of the synthesis filter bank. In practical applications, a popular choice is based on the Haar transforms [19,68].

Haar pre- and postfilters have the advantage of simultaneously possessing symmetry and orthogonality, and no multiplication is needed if one ignores the scaling factor. The prefilters should be as short as possible to preserve the time localization of the wavelet decomposition. The choice of prefilter is often critical for the results, and should be made depending on the application at hand and the type of signal to be processed.

The postfilter $\mathscr{N}(z)$ that accompanies the prefilter $\mathscr{M}(z)$ must satisfy $\mathscr{N}(z) \mathscr{M}(z)=I$. The matrix coefficients of the pre- and postfilters we used are shown in table 3 
Table 3 The matrix coefficients of the pre- and postfilters for GHM, CL, and SA1 orthogonal multiwavelets

\begin{tabular}{ccc}
\hline & $\mathscr{M}(z)$ & $\mathscr{N}(z)$ \\
\hline GHM & $\frac{1}{8 \sqrt{2}}\left(\left[\begin{array}{cc}3 & 10 \\
0 & 0\end{array}\right]+\left[\begin{array}{cc}3 & 0 \\
8 \sqrt{2} & 0\end{array}\right] z^{-1}\right)$ & $\frac{1}{10}\left(\left[\begin{array}{cc}0 & 10 \\
0 & -3\end{array}\right] z+\left[\begin{array}{cc}0 & 0 \\
8 \sqrt{2} & -3\end{array}\right]\right)$ \\
CL & {$\left[\begin{array}{c}1 / 4 \\
1 /(1+\sqrt{7})-1 /(1+\sqrt{7})\end{array}\right]$} & {$\left[\begin{array}{cc}2 & (1+\sqrt{7}) / 2 \\
2 & -(1+\sqrt{7}) / 2\end{array}\right]$} \\
SA1 & $\frac{1}{\sqrt{2}}\left[\begin{array}{cc}1 & 1 \\
1 & -1\end{array}\right]$ & $\frac{1}{\sqrt{2}}\left[\begin{array}{cc}1 & 1 \\
1 & -1\end{array}\right]$ \\
\hline
\end{tabular}

6.3 Dyadic approximation of a 1D signal

As explained in section 5.2, the customer often prefers a simple dyadic approximation of the coefficients. For a chosen $b_{0}$ and $J$, we let $\hat{\mathbf{s}}_{1, J}$ be the signal obtained by decomposing and reconstructing through $J$ levels, using $\left(b_{0}+1\right)$ bits of accuracy. We define the quantization error by the norm

$$
\left\|\varepsilon_{1, J}\right\|_{\infty}=\max _{i}\left|\varepsilon_{1, J}(i)\right|
$$

where

$$
\varepsilon_{1, J}(i)=\mathbf{s}(i)-\hat{\mathbf{s}}_{1, J}(i) .
$$

We mainly will consider balanced and non-balanced multiwavelet decomposition and synthesis without processing with a 2-bit approximation of the coefficient $\sqrt{3}$, i. e. $b_{0}=1$, $\sqrt{3} \approx 3 / 2$.

The non-balanced DMWT converts the scalar input signal into a vector input signal by simple partitioning. The balanced DWMT uses a preprocessing and postprocessing step.

Results of using the SA1 balanced filter bank with the Haar pre- and postfilters given in table 3 for the 'Piece-Regular' signal with $2^{10}$ samples, through 2 and 6 decomposition levels, with their these quantization errors are shown in fig. 6.a), (b). Obviously, increasing the decomposition level leads to an increase in quantization error.

It is important to note that the reconstructed signal follows the input signal, except at spikes where the local maxima of the quantization errors are achieved. The best results are obtained at small decomposition levels, between 1 and 4, as shown in fig. 6(a). Higher decomposition levels lead to an increase of the quantization errors over the input signal; see fig. 6. b).

In contrast to the balanced SA1 multiwavelet, the quantization errors of the non-balanced SA1 multiwavelet are very different, and poor. The reconstructed signal oscillates around the analyzing signal, and the quantization errors are bigger than the balanced version, even at low levels, as shown in fig. 7.a).

Detailed information about the minimal and maximal quantization errors, measured by the norm $\left\|\varepsilon_{1, J}\right\|_{\infty}$ for the test signal 'Piece-Regular' (see fig. 6), and the additional test signal 'Piece-Polynomial' (see fig. 8) with $2^{9}$ to $2^{13}$ samples are tabulated in tables 4 and 5

The minimal quantization errors are at the first decomposition level for all lengths of both test signals, while the maximum errors depend on the test signal. There are only minor differences in the minimal quantization errors for the 'Piece-Regular' signal for all signal lengths, which means that the minimal quantization error is almost independent of the length of this signal. 

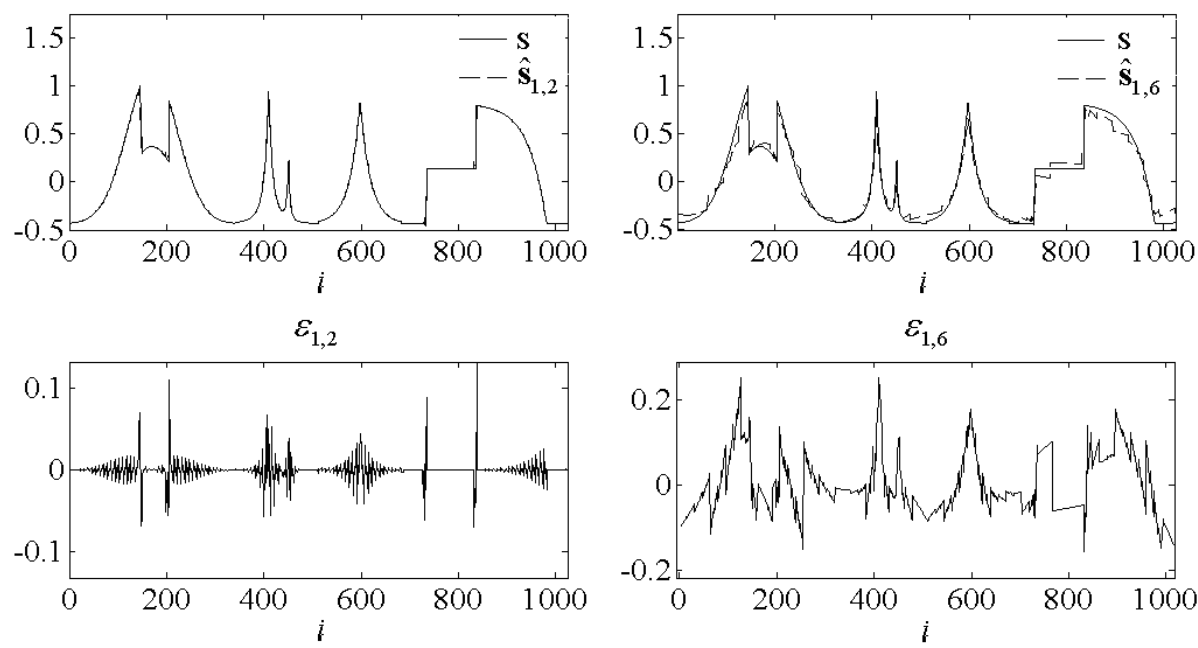

(a)

(b)

Fig. 6 Influence of the number of decomposition levels $J$ of the 2-bit dyadic approximations for the coefficient $\sqrt{3}$ ( i. e. $\sqrt{3} \approx 3 / 2$ ) of the balanced SA1 multiwavelet analysis and synthesis filter bank without processing, for the 'Piece-Regular' signal with $i=2^{10}$ samples through (a) $J=2$ levels with $\left\|\varepsilon_{1,2}\right\|_{\infty}=0.1317$; (b) $J=6$ levels with $\left\|\varepsilon_{1,6}\right\|_{\infty}=0.2535$. In both (a) and (b), the top image shows the original signal $\mathbf{s}$ and reconstruction $\hat{\mathbf{s}}_{1, J}$; the bottom image shows the error $\varepsilon_{1, J}$.

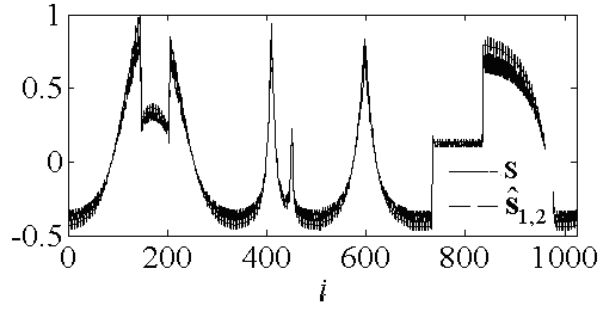

$\varepsilon_{1,2}$

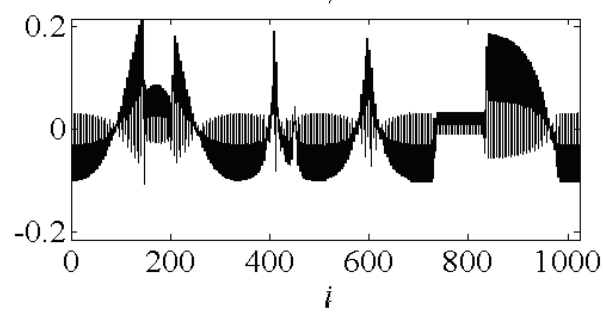

(a)

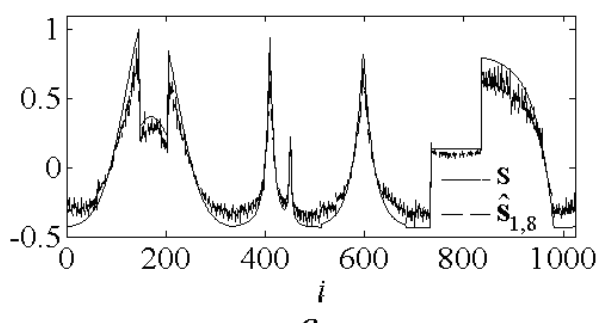

$\varepsilon_{1,8}$

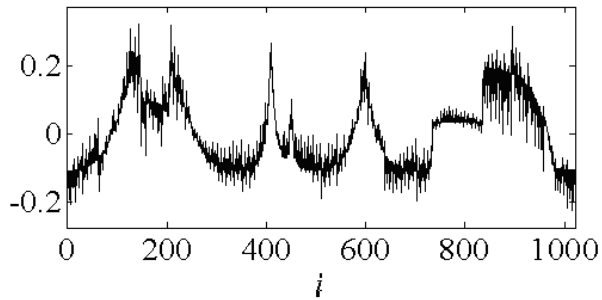

(b)

Fig. 7 Influence of the number of decomposition levels $J$ of the 2-bit dyadic approximations for the coefficient $\sqrt{3}$ ( i. e. $\sqrt{3} \approx 3 / 2$ ) of the non-balanced SA1 multiwavelet analysis and synthesis filter bank without processing, for the 'Piece-Regular' signal with $i=2^{10}$ samples through (a) $J=2$ levels with $\left\|\varepsilon_{1,2}\right\|_{\infty}=0.2148$; (b) $J=8$ levels with $\left\|\varepsilon_{1,8}\right\|_{\infty}=0.3239$. In both (a) and (b), the top image shows the original signal $\mathbf{s}$ and reconstruction $\hat{\mathbf{s}}_{1, J}$; the bottom image shows the error $\varepsilon_{1, J}$. 
Table 4 Dependence of the norm $\left\|\varepsilon_{1, J}\right\|_{\infty}$ on the number of decomposition levels $J$ for the balanced SA1 multiwavelet without processing. The 2-bit dyadic approximation of the coefficient $\sqrt{3}$ (i. e. $\sqrt{3} \approx 3 / 2$ ) is applied to the 'Piece-Regular' signal.

\begin{tabular}{cccccc}
\hline $\begin{array}{c}\text { Decomp. } \\
\text { levels }\end{array}$ & $2^{9}$ samples & $2^{10}$ samples & $2^{11}$ samples & $2^{12}$ samples & $2^{13}$ samples \\
\hline 1 & $\mathbf{0 . 0 6 0 8 4}$ & $\mathbf{0 . 0 6 1 4 9}$ & $\mathbf{0 . 0 6 6 9 5}$ & $\mathbf{0 . 0 6 6 4 6}$ & $\mathbf{0 . 0 6 1 0 3}$ \\
2 & 0.12786 & 0.13167 & 0.12747 & 0.14190 & 0.10010 \\
3 & 0.18903 & 0.13848 & 0.13056 & 0.14656 & 0.13211 \\
4 & 0.23077 & 0.18432 & 0.18191 & 0.17597 & 0.13931 \\
5 & 0.26208 & 0.21887 & 0.16843 & 0.20945 & 0.14795 \\
6 & $\mathbf{0 . 2 9 2 7 7}$ & 0.25346 & 0.21485 & 0.17841 & 0.18582 \\
7 & 0.26932 & $\mathbf{0 . 2 8 6 4 1}$ & 0.26010 & 0.22375 & 0.17793 \\
8 & & 0.26101 & 0.28482 & 0.26315 & 0.22557 \\
9 & & & $\mathbf{0 . 2 9 0 9 9}$ & 0.29428 & 0.26304 \\
10 & & & & $\mathbf{0 . 3 0 9 1 1}$ & $\mathbf{0 . 2 9 5 6 9}$ \\
11 & & & & & 0.28781 \\
\hline
\end{tabular}

Table 5 Dependence of the norm $\left\|\varepsilon_{1, J}\right\|_{\infty}$ on the number of decomposition levels $J$ for the balanced SA1 multiwavelet without processing. The 2-bit dyadic approximation of the coefficient $\sqrt{3}$ (i. e. $\sqrt{3} \approx 3 / 2$ ) is applied to the 'Piece-Polynomial' signal.

\begin{tabular}{cccccc}
\hline $\begin{array}{c}\text { Decomp. } \\
\text { levels }\end{array}$ & $2^{9}$ samples & $2^{10}$ samples & $2^{11}$ samples & $2^{12}$ samples & $2^{13}$ samples \\
\hline 1 & $\mathbf{0 . 0 5 8 3 4}$ & $\mathbf{0 . 1 3 1 2 4}$ & $\mathbf{0 . 1 3 1 1 8}$ & $\mathbf{0 . 1 3 0 7 3}$ & $\mathbf{0 . 0 5 7 7 0}$ \\
2 & 0.13597 & 0.25323 & 0.27918 & 0.27996 & 0.13145 \\
3 & 0.25061 & 0.26580 & 0.38819 & 0.29392 & 0.26348 \\
4 & 0.25272 & $\mathbf{0 . 3 7 8 4 1}$ & 0.32504 & $\mathbf{0 . 3 9 0 6 7}$ & 0.28238 \\
5 & $\mathbf{0 . 3 1 0 7 4}$ & 0.32303 & $\mathbf{0 . 3 7 0 1 6}$ & 0.31828 & $\mathbf{0 . 3 9 4 0 0}$ \\
6 & 0.26599 & 0.30530 & 0.33977 & 0.38265 & 0.33192 \\
7 & 0.26648 & 0.36935 & 0.36768 & 0.33015 & 0.37298 \\
8 & & 0.36903 & 0.36846 & 0.35761 & 0.34802 \\
9 & & & 0.36837 & 0.36052 & 0.37613 \\
10 & & & & 0.36055 & 0.37174 \\
11 & & & & & 0.37168 \\
\hline
\end{tabular}

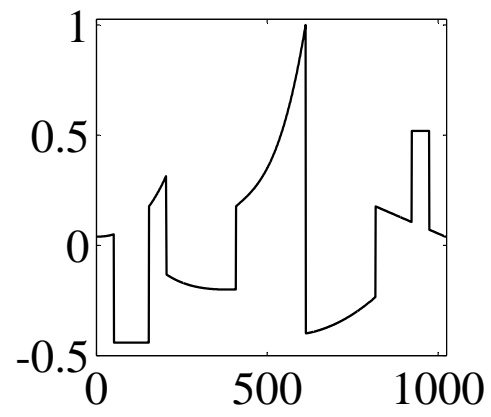

Fig. 8 Additional test signal 'Piece-Polynomial' 
The minimal quantization errors for the 'Piece-Polynomial' signal for signal lengths $2^{10}$ to $2^{12}$ samples are nearly equal, but for $2^{9}$ and $2^{13}$ samples decrease by more than a factor of 2 .

The maximum quantization errors for the 'Piece-Regular' signal are at the first and next to last levels and are nearly equal, and for the 'Piece-Polynomial' signal at decomposition levels 4 or 5 .

Note that the minimal quantization errors are in the range $[0.01,0.05]$, but the maximal quantization errors are in the range $[0.3,0.4]$, which leads to visible artifacts in signal and image processing.

\subsection{Dyadic approximations for 2D signals}

In this subsection, we consider the influence of 2-bit $(\sqrt{3} \approx 3 / 2)$ and 3-bit $(\sqrt{3} \approx 7 / 4)$ dyadic approximation applied to analysis and reconstruction filter bank without processing, in the context of the balanced and non-balanced SA1 multiwavelet. We will consider the gray scale image 'Lena' of size $256 \times 256$ pixels.

The first example illustrates the effect of the 2-bit dyadic approximation of the coefficient $\sqrt{3}$ (i. e. $\sqrt{3} \approx 3 / 2$ ). As we can see see from fig. 9 (a), image processing with the SA1 non-balanced multiwavelet filter bank leads a grid artifact over the whole image. Furthermore, with more than one decomposition level the obtained images are usually unusable. It is also important to note that after more than $J=4$ decomposition levels, the multiwavelet processing with the balanced filter bank shows visible square edge artifacts, even though the PSNR can be high. This is shown in fig. 9 (b).

The second example illustrates a comparison of 3-bit dyadic approximation of the coefficient $\sqrt{3}$ (i.e. $\sqrt{3} \approx 7 / 4$ ). It is obvious from fig. 10 a) and (b) that the SA1 multiwavelet processing with both non-balanced and balanced filter bank leads to high quality images. Therefore, in some applications we prefer 3 -bit dyadic approximation of the coefficient $\sqrt{3}$, which does not lead to undesired image artifacts.

\subsection{Image Denoising}

In order to suppress the noise coefficients, the noisy image is first transformed to the multiwavelet domain, and then soft or hard vector thresholding is applied at various resolution levels. This is described in more detail in subsection 2.2 .

We apply image denoising to the test images shown in fig. 11 and fig. 12, using the lifting scheme (eqs. 20] and (21)) and the multiwavelet decomposition in [69], for three multiwavelets GHM, CL, and SA1. The images are of size $256 \times 256$ or $512 \times 512$ pixels, with additive white Gaussian noise (AWGN) with variance $\sigma=10$. Either soft or hard vector thresholding is applied.

The quality of an image denoising algorithm is determined by a distortion measurement for determining how much information has been lost when the reconstructed image is produced from the denoised images. The most often used measurement is the Mean Square Error (MSE). For an image of size $N \times N$, it is

$$
\operatorname{MSE}=\frac{1}{N^{2}} \sum_{x, y=1}^{N}(s(x, y)-\hat{s}(x, y))^{2},
$$

where $s(x, y)$ is the original image, and $\hat{s}(x, y)$ is the denoised image. 

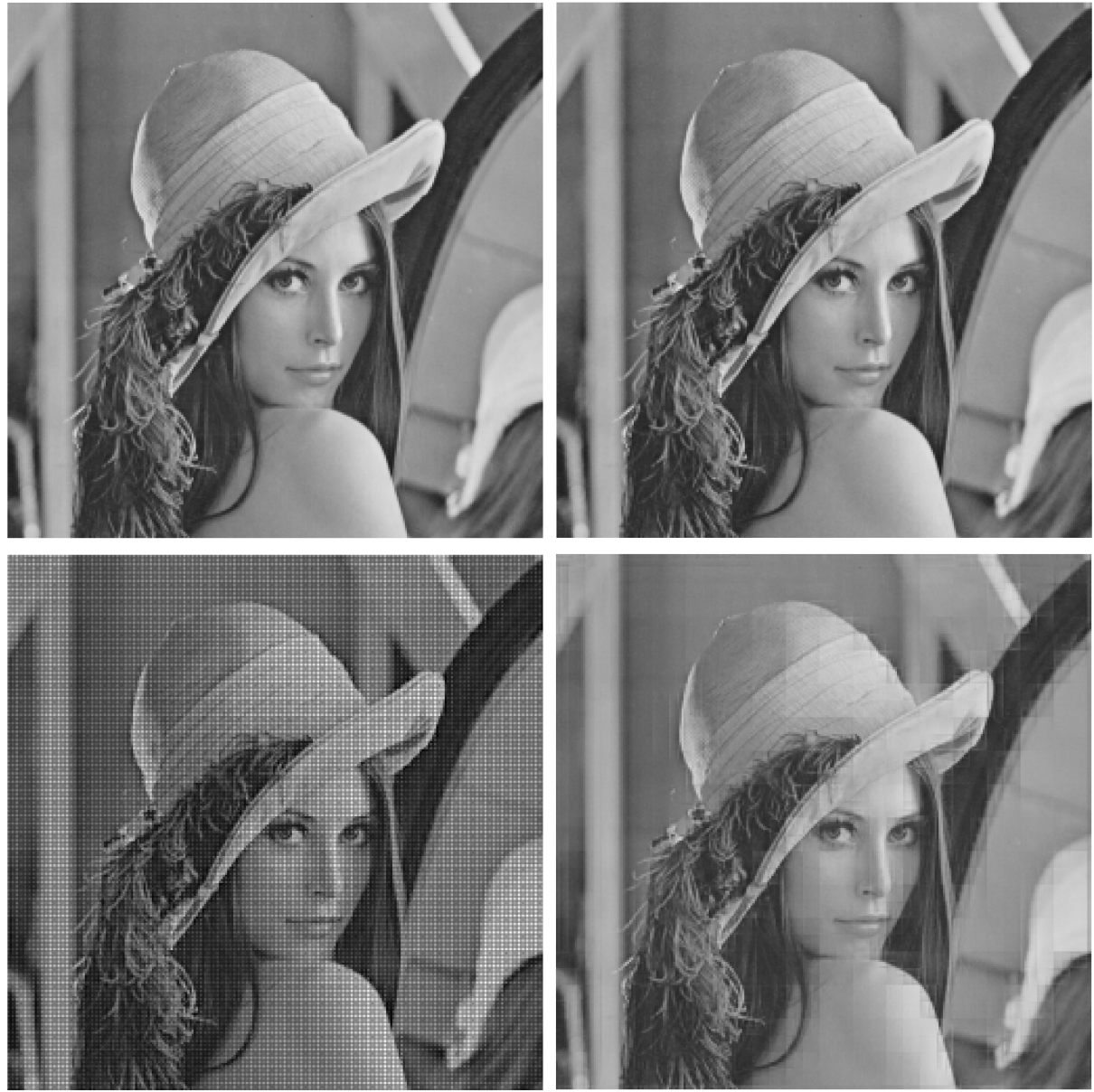

(a)

(b)

Fig. 9 Influence of the number of decomposition levels for 2-bit dyadic approximations for the coefficient $\sqrt{3}$ (i. e. $\sqrt{3} \approx 3 / 2$ ) of the SA1 multiwavelet analysis and synthesis filter bank without processing. (a) Nonbalanced; top: $J=1$ level, PSNR $=\infty$; bottom: $J=4$ levels, PSNR $=17.12 \mathrm{~dB}$. (b) Balanced; top: $J=1$ level, PSNR $=323.35 \mathrm{~dB}$; bottom: $J=4$ levels, PSNR $=30.07 \mathrm{~dB}$.

The Peak Signal-to-Noise Ratio (PSNR) is the MSE in decibels on a logarithmic scale:

$$
\mathrm{PNSR}=10 \log _{10}\left(255^{2} / \mathrm{MSE}\right) \mathrm{dB}
$$

The results shown in tables 6 and 7 provide a comparison of PSNRs (in dB) for AWGN with variance $\sigma=10$. Best results are shown in boldface. Table 6 shows the results for the images of size $256 \times 256$ pixels; table 7 shows corresponding results for the images of size $512 \times 512$ pixels.

In general, the best performance for hard thresholding is provided by SA1, in both tables; for soft thresholding, it is GHM. 

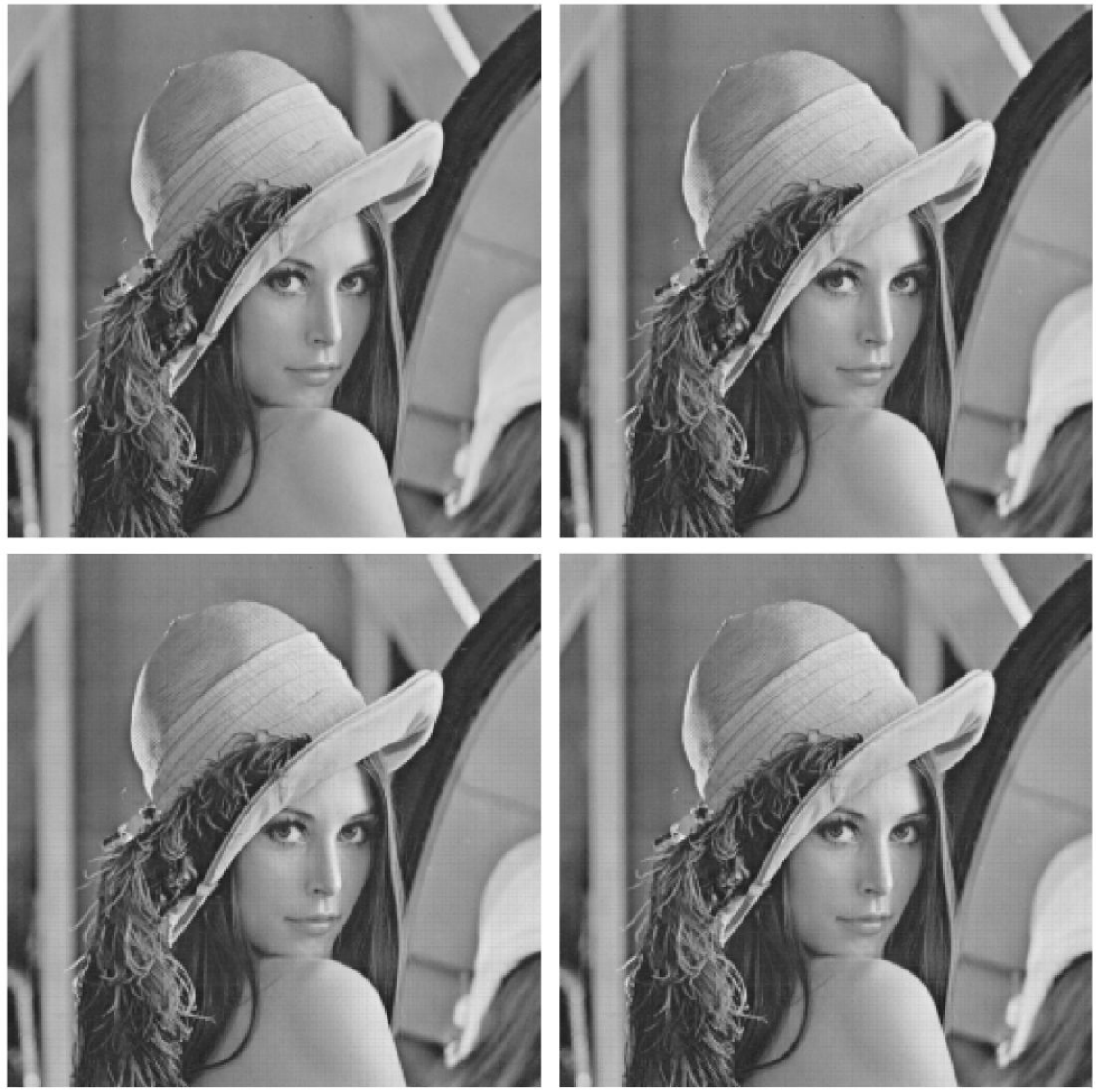

(a)

(b)

Fig. 10 Influence of the number of decomposition levels for 3-bit dyadic approximations for the coefficient $\sqrt{3}$ (i. e. $\sqrt{3} \approx 7 / 4$ ) of the SA1 multiwavelet analysis and synthesis filter bank without processing. (a) Nonbalanced; top: $J=1$ level, PSNR $=\infty$; bottom: $J=4$ levels, PSNR $=38.07 \mathrm{~dB}$. (b) Balanced; top: $J=1$ level, PSNR $=323.35 \mathrm{~dB}$; bottom: $J=4$ levels, PSNR $=50.44 \mathrm{~dB}$.
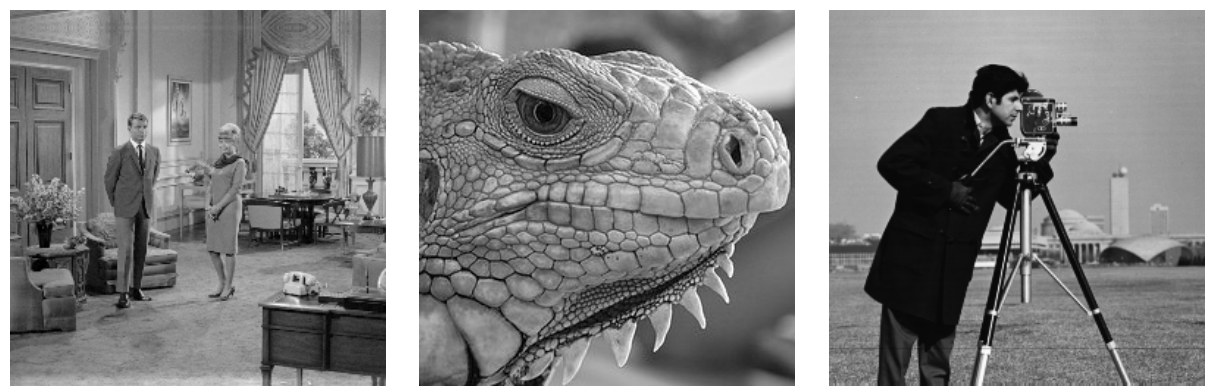

Fig. 11 Test images of size $256 \times 256$ pixels ('Couple', 'Lizard', 'Cameraman'). A $256 \times 256$ version of 'House' was also used (see fig. 12 . 
Table 6 Comparative results of PSNRs of denoised test images for three non-balanced orthogonal multiwavelets (GHM, CL, SA1) through $J=5$ decomposition levels with AWGN (with variance $\sigma=10$ ) and vector thresholds. The test images are of size $256 \times 256$ pixels.

\begin{tabular}{|c|c|c|c|c|c|c|c|c|}
\hline 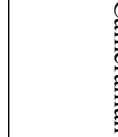 & & & & & & & & 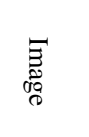 \\
\hline 总 & $\stackrel{\mathscr{O}}{\Rightarrow}$ & 呇 & $\stackrel{\circ}{g}$ & 言 & $\stackrel{\circ}{\Rightarrow}$ & 总 & $\stackrel{\mathscr{g}}{g}$ & $\frac{\mathscr{D}}{\frac{0}{0}}$ \\
\hline 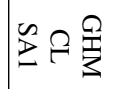 & $\stackrel{2}{2} \beta$ & $\geqslant \rho \frac{\Omega}{3}$ & $\tilde{D} \rho$ & $\theta \rho \frac{8}{3}$ & $\sum \rho$ & $\sum \beta \frac{\Omega}{3}$ & $\stackrel{n}{\geq} \cap \frac{2}{3}$ & 层 \\
\hline 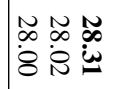 & 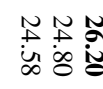 & 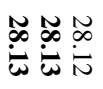 & 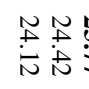 & 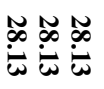 & 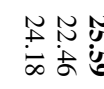 & 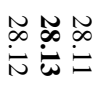 & 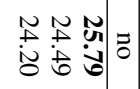 & \\
\hline 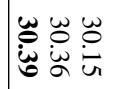 & 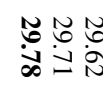 & 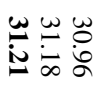 & $\underset{\omega}{\omega} \stackrel{\omega}{\omega}$ & 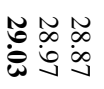 & 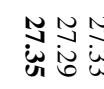 & 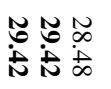 & 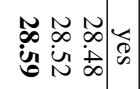 & \\
\hline 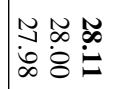 & 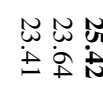 & 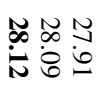 & 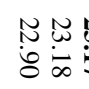 & 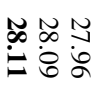 & 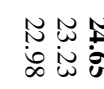 & 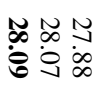 & 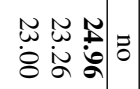 & \\
\hline 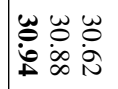 & 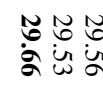 & 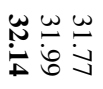 & 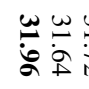 & 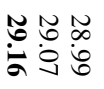 & $\begin{array}{l}\tilde{\alpha} \tilde{\alpha}= \\
\dot{\alpha} \dot{a}=\end{array}$ & 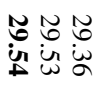 & 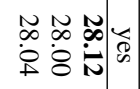 & \\
\hline 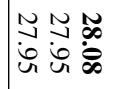 & 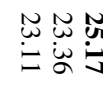 & 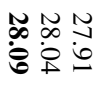 & 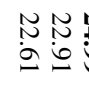 & 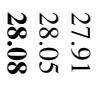 & 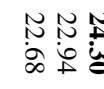 & 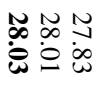 & 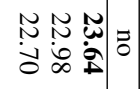 & \\
\hline 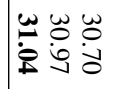 & 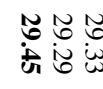 & 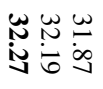 & 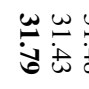 & 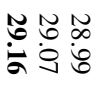 & 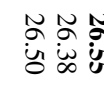 & 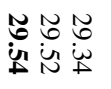 & 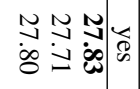 & \\
\hline 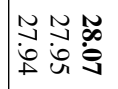 & 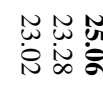 & 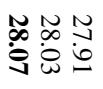 & 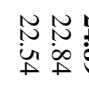 & 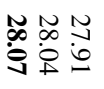 & 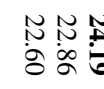 & 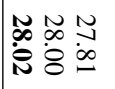 & 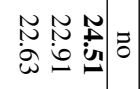 & \\
\hline 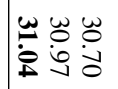 & 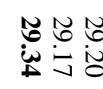 & 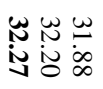 & 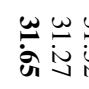 & 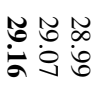 & 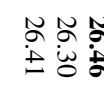 & 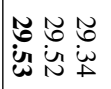 & 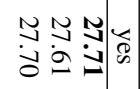 & \\
\hline 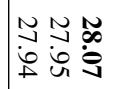 & 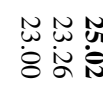 & 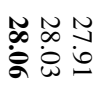 & 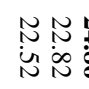 & 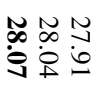 & 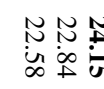 & 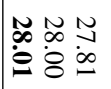 & $\mid$ & \\
\hline 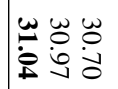 & 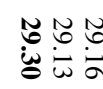 & 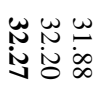 & 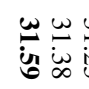 & 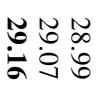 & 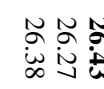 & 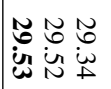 & 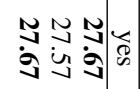 & \\
\hline
\end{tabular}


Table 7 Comparative results of PSNRs of denoised test images for three non-balanced orthogonal multiwavelets (GHM, CL, SA1) through $J=5$ decomposition levels with AWGN (with variance $\sigma=10$ ) and vector thresholds. The test images are of size $512 \times 512$ pixels.

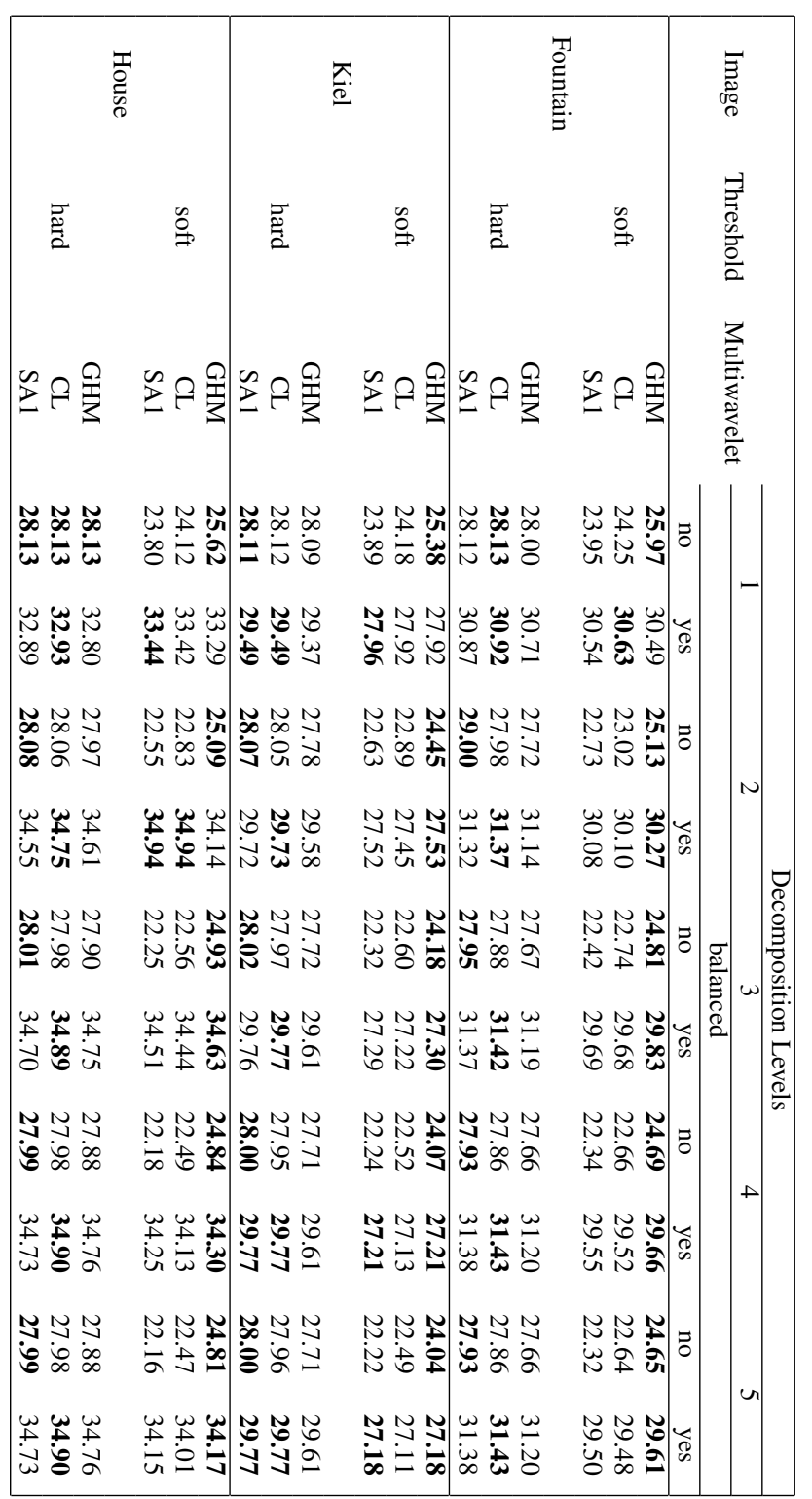



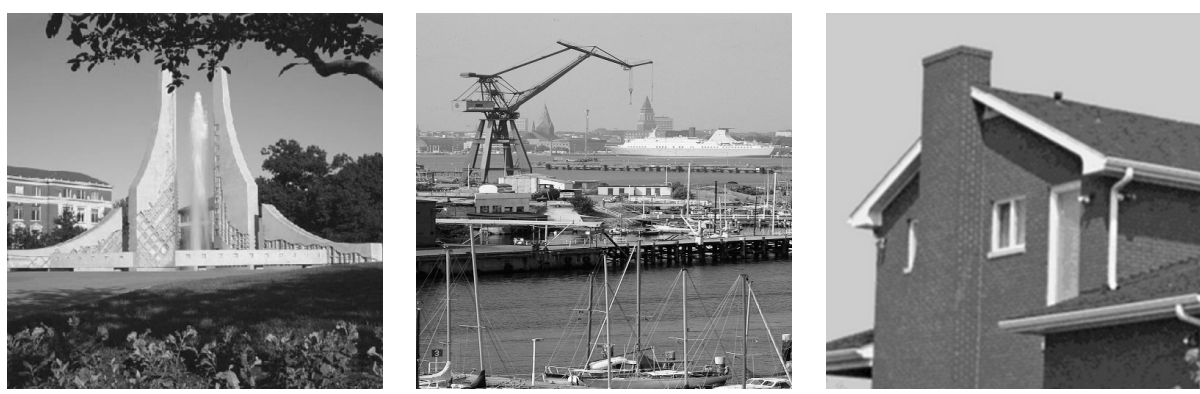

Fig. 12 Test images of size $512 \times 512$ pixels ('Fountain', 'Kiel', 'House').

\section{Conclusion}

We have presented a novel mathematical algorithm for simple orthogonal multiwavelet construction and a comprehensive design approach for spectral matrix decomposition and efficient lifting scheme implementation. We designed a simple orthogonal and regular multiwavelet with symmetry properties. The matrix spectral factorization algorithm of Youla and Kazanjian was used, effectively increasing its precision. It can be used in the future to find new exact supercompact multiwavelets with better regularity and CG. The coding gain of the design is very good, especially considering its simplicity. The obtained multiwavelet SA1 can be used in denoising image processing as well other applications in communication systems. By using the property that the inverse matrix of an integer triangular matrix is also an integer triangular matrix, up to a scalar factor, a lifting scheme based on a PLUS type matrix decomposition was constructed. An important note for further work is that the lifting scheme is a proper choice, as in the present work. We have shown that the proposed lifting scheme with reduced accuracy of the present SA1 multiwavelet is also capable of denoising images at different decomposition levels, and can be used for image denoising and reversible integer to integer multiwavelet transforms.

Acknowledgements The authors would like to thank the anonymous reviewers for their careful reading and helpful suggestions.

\section{References}

1. Abbas, Z., Vahdati, S., Mohd Atan, K.A., Nik Long, M.A.: Legendre multi-wavelets direct method for linear integro-differential equations. Applied Mathematical Sciences 3, 693-700 (2009)

2. Alpert, B.K.: Sparse representation of smooth linear operators. Tech. Rep. YALEU/DCS/RR-814, Yale University, New Haven, CT (1990)

3. Averbuch, A.Z., Zheludev, V.A., Cohen, T.: Multiwavelet frames in signal space originated from Hermite splines. IEEE Trans. Signal Process. 55(3), 797-808 (2007)

4. Bacchelli, S., Papi, S.: Matrix thresholding for multiwavelet image denoising. Numerical Algorithms 33, 41-52 (2003)

5. Baran, Á., Stoyan, G.: Gauss-Legendre elements: a stable, higher order non-conforming finite element family. Computing 79(1), 1-21 (2007)

6. Bauer, F.L.: Beiträge zur Entwicklung numerischer Verfahren für programmgesteuerte Rechenanlagen. II. Direkte Faktorisierung eines Polynoms. Bayer. Akad. Wiss. Math.-Nat. Kl. S.-B. 1956, 163-203 (1956)

7. Beam, R.M., Warming, R.F.: Multiresolution analysis and supercompact multiwavelets. SIAM Journal on Scientific Computing 22(4), 1238-1268 (2000) 
8. Bhati, D., Pachori, R.B., Sharma, M., Gadre, V.M.: Design of time-frequency-localized two-band orthogonal wavelet filter banks. Circuits, Systems, and Signal Processing 37(8), 3295-3312 (2018)

9. Bose, N.K.: Applied Multidimensional Systems Theory, 2nd edn. Springer International Publishing, Basel (2017)

10. Bruce, A.G., Gao, H.Y.: Understanding WaveShrink: Variance and bias estimation. Biometrika 83(4), 727-745 (1996)

11. Calderbank, A., Daubechies, I., Sweldens, W., Yeo, B.L.: Wavelet transforms that map integers to integers. Applied and Computational Harmonic Analysis 5(3), 332-369 (1998)

12. Callier, F.M.: On polynomial spectral factorization by symmetric extraction. IEEE Trans. Automat. Control 30(5), 453-464 (1985)

13. Carathéodory, C.: Über den Variabilitätsbereich der Koeffizienten von Potenzreihen, die gegebene Werte nicht annehmen. Mathematische Annalen 64, 95-115 (1907)

14. Charoenlarpnopparut, C.: One-dimensional and multidimensional spectral factorization using Gröbner basis approach. In: 2007 Asia-Pacific Conference on Communications, pp. 201-204 (2007)

15. Cheung, K.W., Po, L.M.: Integer multiwavelet transform for lossless image coding. In: Proc. 2001 International Symposium on Intelligent Multimedia, Video and Speech Processing, pp. 117-120 (2001)

16. Chui, C.K., Lian, J.A.: A study of orthonormal multi-wavelets. Appl. Numer. Math. 20(3), 273-298 (1996)

17. Cooklev, T.: Regular perfect-reconstruction filter banks and wavelet bases. Ph.D. thesis, Tokyo University of Technology, Tokyo, Japan (1995)

18. Cooklev, T., Nishihara, A., Kato, M., Sablatash, M.: Two-channel multifilter banks and multiwavelets. In: 1996 IEEE International Conference on Acoustics, Speech, and Signal Processing Conference Proceedings, vol. 5, pp. 2769-2772 (1996)

19. Cotronei, M., Montefusco, L.B., Puccio, L.: Multiwavelet analysis and signal processing. IEEE Transactions on Circuits and Systems II: Analog and Digital Signal Processing 45(8), 970-987 (1998)

20. Daubechies, I., Guskov, I., Schröder, P., Sweldens, W.: Wavelets on irregular point sets. Philosophical Transactions of the Royal Society of London A: Mathematical, Physical and Engineering Sciences 357(1760), 2397-2413 (1999). DOI 10.1098/rsta.1999.0439

21. Dechevsky, L.T., Grip, N., Gundersen, J.: A new generation of wavelet shrinkage: adaptive strategies based on composition of Lorentz-type thresholding and Besov-type non-thresholding shrinkage. In: F. Truchetet, O. Laligant (eds.) Proc. SPIE 6763, Wavelet Applications in Industrial Processing V, Boston, MA, USA, pp. 1-14 (2007)

22. Dechevsky, L.T., Gundersen, J., Grip, N.: Wavelet compression, data fitting and approximation based on adaptive composition of Lorentz-type thresholding and Besov-type non-threshold shrinkage. In: I. Lirkov, S. Margenov, J. Waśniewski (eds.) Large-Scale Scientific Computing, 7th International Conference, LSSC 2009, Sozopol, Bulgaria, June 4-8, 2009. Revised Papers, vol. 5910, pp. 738-746. Springer, Heidelberg (2009)

23. Devi, M., Verma, S.R.: An evaluation of system non-homogeneous differential equations using linear Legendre multiwavelet. Indian Journal of Mathematics and Mathematical Sciences 13(1), 243-254 (2017)

24. Donoho, D.L.: De-noising by soft-thresholding. IEEE Trans. Inform. Theory 41(3), 613-627 (1995)

25. Donoho, D.L., Johnstone, I.M.: Ideal spatial adaption via wavelet shrinkage. Biometrika 81(3), 425-455 (1994)

26. Donoho, D.L., Johnstone, I.M.: Minimax estimation via wavelet shrinkage. Ann. Statist. 26(3), 879-921 (1998)

27. Donoho, D.L., Johnstone, I.M., Kerkyacharian, G., Picard, D.: Wavelet shrinkage: Asymptopia? Journal of the Royal Statistical Society. Series B (Methodological) 57(2), 301-369 (1995)

28. Downie, T.R., Silverman, B.W.: The discrete multiple wavelet transform and thresholding methods. IEEE Transactions on Signal Processing 46(9), 2558-2561 (1998)

29. Dritschel, M.A.: On factorization of trigonometric polynomials. Integral Equations and Operator Theory 49(1), 11-42 (2004)

30. Du, B., Xu, X., Dai, X.: Minimum-phase FIR precoder design for multicasting over MIMO frequencyselective channels. Journal of Electronics (China) 30(4), 319-327 (2013)

31. Durand, S., Froment, J.: Artifact free signal denoising with wavelets. In: IEEE International Conference on Acoustics, Speech, and Signal Processing (ICASSP, vol. 6, pp. 3685-3688 (2001)

32. Ephremidze, L., Janashia, G., Lagvilava, E.: A new efficient matrix spectral factorization algorithm. In: SICE Annual Conference 2007, pp. 20-23 (2007)

33. Ephremidze, L., Saied, F., Spitkovsky, I.: On the algorithmization of Janashia-Lagvilava matrix spectral factorization method. IEEE Transactions on Information Theory 64(2), 728-737 (2018)

34. Fejér, L.: Über trigonometrische Polynome. J. Reine Angew. Math. (Crelles Journal) 146, 53-82 (1916) 
35. Fischer, R.F.H.: Sorted spectral factorization of matrix polynomials in MIMO communications. IEEE Transactions on Communications 53(6), 945-951 (2005)

36. Gan, L., Ma, K.K.: On minimal lattice factorizations of symmetric-antisymmetric multifilterbanks. IEEE Transactions on Signal Processing 53(2), 606-621 (2005)

37. Geronimo, J.S., Hardin, D.P., Massopust, P.R.: Fractal functions and wavelet expansions based on several scaling functions. J. Approx. Theory 78(3), 373-401 (1994)

38. Geronimo, J.S., Woerdeman, H.J.: Positive extensions, Fejér-Riesz factorization and autoregressive filters in two variables. Annals of Mathematics 160(3), 839-906 (2004)

39. Hansen, M., Christensen, L.P.B., Winther, O.: Computing the minimum-phase filter using the QLfactorization. IEEE Transactions on Signal Processing 58(6), 3195-3205 (2010)

40. Hao, P., Shi, Q.: Matrix factorizations for reversible integer mapping. IEEE Transactions on Signal Processing 49(10), 2314-2324 (2001)

41. Hornbeck, R.: Numerical methods. QPI series. Quantum Publishers (1975)

42. Janashia, G., Lagvilava, E., Ephremidze, L.: A new method of matrix spectral factorization. IEEE Trans. Inform. Theory 57(4) (2011)

43. Jiang, Q.: On the regularity of matrix refinable functions. SIAM J. Math. Anal. 29(5), 1157-1176 (1998)

44. Jing, M., Huang, H., Liu, W., Qi, C.: A general approach for orthogonal 4-tap integer multiwavelet transforms. Mathematical Problems in Engineering 2010, 1-12 (2010)

45. Kalathil, S., Elias, E.: Prototype filter design approaches for near perfect reconstruction cosine modulated filter banks - a review. Journal of Signal Processing Systems 81(2), 183-195 (2015)

46. Malyshev, A.N.: On the acceleration of an algorithm for polynomial factorization. Doklady Mathematics 88(2), 586-589 (2013)

47. Malyshev, A.N., Sadkane, M.: The Bauer-type factorization of matrix polynomials revisited and extended. Computational Mathematics and Mathematical Physics 58(7), 1025-1034 (2018)

48. Mohamed, M., Torky, M.: Solution of linear system of partial differential equations by Legendre multiwavelet and Chebyshev multiwavelet. International Journal of Scientific and Innovative Mathematical Research 2(12), 966-976 (2014)

49. Moir, T.J.: Toeplitz matrices for LTI systems, an illustration of their application to Wiener filters and estimators. International Journal of Systems Science 49(4), 800-817 (2018)

50. Niu, Y., Shen, L.: Wavelet denoising using the Pareto optimal threshold. International Journal of Computer Science and Network Security (2007)

51. Prandoni, P., Vetterli, M.: Approximation and compression of piecewise smooth functions. Philosophical Transactions: Mathematical, Physical and Engineering Sciences 357(1760), 2573-2591 (1999)

52. Riesz, F.: Über ein Problem des Herrn Carathéodory. J. Reine Angew. Math (Crelles Journal) 146, 83-87 (1916)

53. Rioul, O., Vetterli, M.: Wavelets and signal processing. IEEE Signal Processing Magazine 8(4), 14-38 (1991)

54. Rudin, W.: The extension problem for positive-definite functions. Illinois J. Math. 7(3), 532-539 (1963)

55. Sadov, S.Y., McGreer, K.A.: Legendre polynomials as finite elements in boundary integral equations for transmission problem with periodic piecewise-linear boundary. In: DIPED - 99. Direct and Inverse Problems of Electromagnetic and Acoustic Wave Theory. Proceedings of 4th International Seminar/Workshop (IEEE Cat. No.99TH8402), pp. 55-58 (1999)

56. She, Y., Hao, P.: On the necessity and sufficiency of PLUS factorizations. Linear Algebra and its Applications 400, 193-202 (2005)

57. Shen, L., Tan, H.H., Tham, J.Y.: Symmetric-antisymmetric orthonormal multiwavelets and related scalar wavelets. Appl. Comput. Harmon. Anal. 8(3), 258-279 (2000)

58. Smith, C.B., Smith, C.B., Agaian, S., Akopian, D.: A wavelet-denoising approach using polynomial threshold operators. IEEE Signal Processing Letters 15, 906-909 (2008)

59. Smith, M., Barnwell, T.: Exact reconstruction techniques for tree-structured subband coders. IEEE Transactions on Acoustics, Speech, and Signal Processing 34(3), 434-441 (1986)

60. Soman, A.K., Vaidyanathan, P.P.: Coding gain in paraunitary analysis/synthesis systems. IEEE Transactions on Signal Processing 41(5), 1824-1835 (1993)

61. Soman, K.P., Ramachandran, K.I.: Insight into wavelets: From theory to practice. Prentice-Hall of India, New Delhi (2004)

62. Strang, G.: Linear Algebra and its Applications. Cengage Learning (2006)

63. Strang, G., Nguyen, T.: Wavelets and filter banks. Wellesley-Cambridge Press, Wellesley, MA (1996)

64. Strang, G., Strela, V.: Orthogonal multiwavelets with vanishing moments. J. Optical Engineering 33(7), 2104-2107 (1994)

65. Strang, G., Strela, V.: Short wavelets and matrix dilation equations. IEEE Transactions on Signal Processing 43(1), 108-115 (1995) 
66. Strela, V.: A note on construction of biorthogonal multi-scaling functions. In: Wavelets, Multiwavelets, and their Applications (San Diego, CA, 1997), Contemp. Math., vol. 216, pp. 149-157. Amer. Math. Soc., Providence, RI (1998)

67. Sweldens, W.: The lifting scheme: a construction of second generation wavelets. SIAM J. Math. Anal. 29(2), 511-546 (1998)

68. Tan, H.H., Shen, L.X., Tham, J.Y.: New biorthogonal multiwavelets for image compression. Signal Processing 79(1), 45-65 (1999)

69. Tham, J.Y., Shen, L., Lee, S.L., Tan, H.H.: A general approach for analysis and application of discrete multiwavelet transforms. IEEE Trans. Signal Process. 48(2), 457-464 (2000)

70. Thyagarajan, K.: Digital Image Processing with Application to Digital Cinema. Focal (2006)

71. Vaidyanathan, P.P.: Theory of optimal orthonormal subband coders. IEEE Transactions on Signal Processing 46(6), 1528-1543 (1998)

72. Vetterli, M.: Filter banks allowing perfect reconstruction. Signal Processing 10(3), 219-244 (1986)

73. Vostry, Z.: A numerical method of matrix spectral factorization. Kybernetika 8(5), 448-470 (1972)

74. Vuik, M.J.: Multiwavelets and outlier detection for troubled-cell indication in discontinuous Galerkin methods. Ph.D. thesis, Delft University of Technology, Institute of Applied Mathematics (2014)

75. Vuik, M.J., Ryan, J.K.: Automated parameters for troubled-cell indicators using outlier detection. SIAM Journal on Scientific Computing 38(1), A84-A104 (2016)

76. Wang, L., Wu, J., Jiao, L., Shi, G.: Lossy-to-lossless hyperspectral image compression based on multiplierless reversible integer TDLT/KLT. IEEE Geoscience and Remote Sensing Letters 6(3), 587-591 (2009)

77. Wang, N., Ge, S., Li, B., Peng, L.: Multiple description image compression based on multiwavelets. International Journal of Wavelets, Multiresolution and Information Processing 17(01), 1850,063-1 1850,063-22 (2019)

78. Wang, Z., McWhirter, J.G., Weiss, S.: Multichannel spectral factorization algorithm using polynomial matrix eigenvalue decomposition. In: 2015 49th Asilomar Conference on Signals, Systems and Computers, pp. 1714-1718 (2015)

79. Wasin, S., Jianzhong, W.: Estimating the support of a scaling vector. SIAM J. Matrix Analysis and Applications 18(1), 63-73 (1997)

80. Whipple, F.J.W.: On the behaviour at the poles of a series of Legendre's functions representing a function with infinite discontinuities. Proceedings of the London Mathematical Society s2-8(1), 213-222 (1910)

81. Yin, S.S., Zhou, Y., Chan, S.C.: An efficient method for designing of modulated filter banks with causalstable IIR filters. Journal of Signal Processing Systems 78(2), 187-197 (2015)

82. Youla, D.C., Kazanjian, N.N.: Bauer-type factorization of positive matrices and the theory of matrix polynomials orthogonal on the unit circle. IEEE Trans. Circuits and Systems CAS-25(2), 57-69 (1978)

83. Zhang, Y., He, N., Zhen, X., Sun, X.: Image denoising based on the wavelet semi-soft threshold and total variation. In: 2017 International Conference on Vision, Image and Signal Processing (ICVISP), pp. 55-62 (2017) 